\title{
Catalytic Asymmetric Synthesis with Rh-Diene Complexes: 1,4-Addition of Arylboronic Acids to Unsaturated Esters
}

\author{
Jean-François Paquin, Corey R. J. Stephenson, Christian Defieber \\ and Erick M. Carreira*
}

The following includes general experimental procedures, specific details for representative reactions, and isolation and spectroscopic information for the new compounds prepared. All reactions were performed in oven dried glassware under argon. ${ }^{1} \mathrm{H}$ and ${ }^{13} \mathrm{C}$ NMR spectra were recorded on a VARIAN Mercury $300 \mathrm{MHz}$ or a Gemini 300 MHz. Infrared spectra were recorded on a Perkin-Elmer RX-I FT-IR or a Perkin Elmer spectrometer. High resolution mass spectra were obtained on a VG-TRIBRID for electron impact ionization (EI). Enantiomeric excesses were determined by chiral HPLC analysis with a Merck-Hitachi D-7000 system. Optical rotation $[\alpha]_{\mathrm{D}}$ were measured on a Jasco DIP-1000 Polarimeter. The absolute stereochemistry was assigned based on the established stereochemical outcome of the reaction of aryl boronic acids with enones ${ }^{1}$ and enals. ${ }^{2}$ The ligands were prepared as previously reported. ${ }^{1,2}$ Ethyl cinnamate (1) and benzyl cinnamate (2) were purchased and used as received. $t$-Butylcinnamates were prepared from the reaction of the corresponding acid with $\mathrm{Boc}_{2} \mathrm{O}$ and DMAP in THF using conditions previously reported. ${ }^{3}$ The spectral data for $t$-butylcinnamate (3), ${ }^{4} t$-butyl

\footnotetext{
${ }^{1}$ Defieber, C.; Paquin, J.-F.; Serna, S.; Carreira, E. M. Org. Lett. 2004, 6, 3873.

${ }^{2}$ Paquin, J.-F.; Defieber, C.; Stephenson, C. R. J.; Carreira, E. M. J. Am. Chem. Soc. 2005, 127, In Press.

3 Takeda, K.; Akiyama, A.; Nakamura, H.; Takizawa, S.-i.; Mizuno, Y.; Takayanagi, H.; Harigaya, Y. Synthesis 1994, 1063.
} 
4-methoxycinnamate, ${ }^{5} t$-butyl 3-fluorocinnamate, ${ }^{6}$ t-butyl 3-(furan-2-yl)acrylate, ${ }^{7}$ and $t$ -

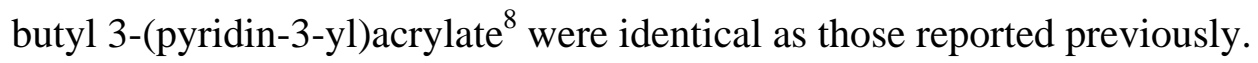

\section{Preparation of t-Butyl Esters}<smiles>CC(C)(C)OC(=O)/C=C/c1ccccc1[N+](=O)[O-]</smiles>

\section{tert-Butyl 3-(2-nitrophenyl)acrylate}

To a solution of 2-nitrocinnamic acid $(1.00 \mathrm{~g}, 5.18 \mathrm{mmol})$ in dry THF $(25.0 \mathrm{~mL})$ was added $\mathrm{Boc}_{2} \mathrm{O}$ (2.26 g, $10.4 \mathrm{mmol}$ ) and DMAP (190 mg, $1.55 \mathrm{mmol}$ ). The reaction mixture was stirred for $72 \mathrm{~h}$ and concentrated. The residue was purified by chromatography on $\mathrm{SiO}_{2}(85: 15$, pentane/Et $2 \mathrm{O})$ to give $1.29 \mathrm{~g}, 100 \%$ of a light yellow solid. IR (neat) $v=2979,2933,1711,1638,1572,1526,1368,1345,1326,1295,1208$, $1153 \mathrm{~cm}^{-1} ;{ }^{1} \mathrm{H}$ NMR (300 MHz, $\left.\mathrm{CDCl}_{3}\right) \delta$ 8.03-7.98 (m, $\left.2 \mathrm{H}\right)$, 7.64-7.63 (m, $\left.2 \mathrm{H}\right)$, 7.567.48 (m, $1 \mathrm{H}), 6.30$ (d, $1 \mathrm{H}, J=15.6 \mathrm{~Hz}), 1.54$ (s, $9 \mathrm{H}) ;{ }^{13} \mathrm{C} \mathrm{NMR}\left(75.5 \mathrm{MHz}, \mathrm{CDCl}_{3}\right) \delta$ 164.8, 148.1, 138.5, 133.2, 130.6, 129.9, 128.9, 125.1, 124.7, 81.1, 28.2; HRMS-EI calcd for $\mathrm{C}_{9} \mathrm{H}_{6} \mathrm{NO}_{3}\left[\mathrm{M}-\mathrm{OC}_{4} \mathrm{H}_{9}\right]^{+}$176.0343, found 176.0343 .

\footnotetext{
${ }^{4}$ Yang, D.; Chen, Y.-C.; Zhu, N.-Y. Org. Lett. 2004, 6, 1577.

${ }^{5}$ Imashiro, R.; Seki, M. J. Org. Chem. 2004, 69, 4216.

${ }^{6}$ Liu, J.; Zhao, Y.; Zhou, Y.; Li, L.; Zhang, T. Y.; Zhang, H. Org. Biomol. Chem. 2003, 1, 3227.

${ }^{7}$ Huang, Z.-Z.; Ye, S.; Xia, W.; Yu, Y.-H.; Tang, Y. J. Org. Chem. 2002, 67, 3096.

${ }^{8}$ Bull, S. D.; Davies, S. G.; Fox, D. J.; Gianotti, M.; Kelly, P. M.; Pierres, C.; Savory, E. D.; Smith, A. D. J. Chem. Soc. Perkin Trans. 1 2002, 16, 1858.
} 


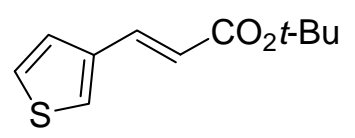

\section{tert-Butyl 3-(thiophen-3-yl)acrylate}

Yellow oil; IR (neat) $v=3099,2976,2932,1699,1632,1366,1309,1279,1141,976$, 854, $782 \mathrm{~cm}^{-1}$; ${ }^{1} \mathrm{H}$ NMR (300 MHz, $\left.\mathrm{CDCl}_{3}\right) \delta 7.57$ (d, $\left.1 \mathrm{H}, J=15.9 \mathrm{~Hz}\right), 7.46(\mathrm{~m}, 1 \mathrm{H})$, 7.35-7.26 (m, 2H), 6.19 (d, 1H, $J=15.9 \mathrm{~Hz}), 1.53(\mathrm{~s}, 9 \mathrm{H}) ;{ }^{13} \mathrm{C}$ NMR $\left(75.5 \mathrm{MHz}, \mathrm{CDCl}_{3}\right)$ $\delta$ 166.4, 137.6, 137.0, 127.4, 126.7, 125.1, 119.8, 80.4, 28.3; HRMS-EI calcd for $\mathrm{C}_{11} \mathrm{H}_{14} \mathrm{O}_{2} \mathrm{~S}[\mathrm{M}]^{+}$210.0715, found 210.0710.

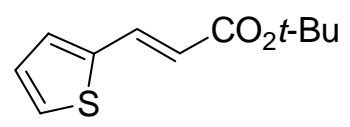

\section{tert-Butyl-3-(thiophen-2-yl)acrylate}

Yellow oil; IR (neat) $v=3106,2977,2931,1703,1626,1427,1391,1367,1310,1283$, 1230, 1208, 971, $852 \mathrm{~cm}^{-1} ;{ }^{1} \mathrm{H}$ NMR (300 MHz, $\left.\mathrm{CDCl}_{3}\right) \delta 7.68$ (d, $\left.1 \mathrm{H}, J=15.6 \mathrm{~Hz}\right), 7.33$ (m, 1H), 7.22 (m, 2H), 7.05-7.02 (m, 1H), 6.17 (d, 1H, $J=15.6 \mathrm{~Hz}), 1.52(\mathrm{~s}, 9 \mathrm{H}) ;{ }^{13} \mathrm{C}$ NMR (75.5 MHz, $\left.\mathrm{CDCl}_{3}\right) \delta$ 166.0, 139.7, 135.9, 130.3, 127.8, 118.9, 80.4, 28.1; HRMSEI calcd for $\mathrm{C}_{11} \mathrm{H}_{14} \mathrm{O}_{2} \mathrm{~S}[\mathrm{M}]^{+}$210.0715, found 210.0712.

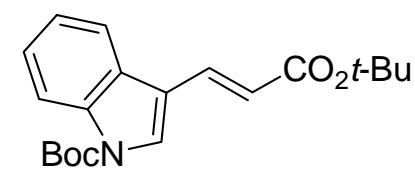

tert-Butyl 3-(3-tert-butoxy-3-oxoprop-1-enyl)-1H-indole-1-carboxylate

Colorless solid; $\mathrm{mp}=111-112{ }^{\circ} \mathrm{C}$; IR (neat) $v=3140,2978,2932,1740,1704,1636$, 1452, 1367, 1258, 1256, 1146, 1094, 913, $743 \mathrm{~cm}^{-1} ;{ }^{1} \mathrm{H}$ NMR (300 MHz, $\left.\mathrm{CDCl}_{3}\right) \delta 8.19$ (d, $1 \mathrm{H}, J=7.8 \mathrm{~Hz}), 7.86-7.83(\mathrm{~m}, 2 \mathrm{H}), 7.73(\mathrm{dd}, 1 \mathrm{H}, J=16.2,0.6 \mathrm{~Hz}), 7.41-7.29(\mathrm{~m}$, 2H), 6.47 (d, $1 \mathrm{H}, J=16.2 \mathrm{~Hz}), 1.68(\mathrm{~s}, 9 \mathrm{H}), 1.53(\mathrm{~s}, 9 \mathrm{H}) ;{ }^{13} \mathrm{C} \mathrm{NMR}\left(75.5 \mathrm{MHz}, \mathrm{CDCl}_{3}\right) \delta$ 
166.6, 149.0, 136.1, 135.1, 128.1, 127.9, 125.1, 123.4, 120.2, 119.4, 116.8, 115.4, 84.5, 80.4, 28.4, 28.3; HRMS-MALDI calcd for $\left[\mathrm{C}_{20} \mathrm{H}_{25} \mathrm{NO}_{4} \mathrm{Na}\right]^{+}$366.1676, found 366.1669.

\section{1,4-Addition to Cinnamates Derivatives - Ligand Screening and Reaction Optimization}

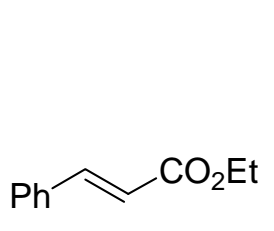

1

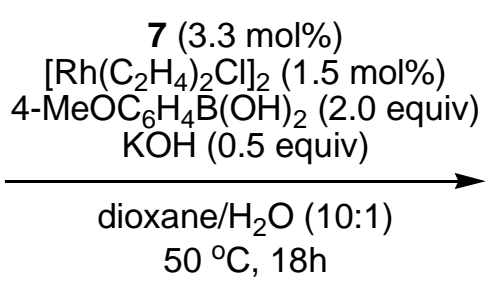

$50{ }^{\circ} \mathrm{C}, 18 \mathrm{~h}$

(S)-Ethyl 3-(4-methoxyphenyl)-3-phenylpropanoate (4). General Procedure in dioxane.

To $\left[\mathrm{Rh}\left(\mathrm{C}_{2} \mathrm{H}_{4}\right) \mathrm{Cl}\right]_{2}(1.7 \mathrm{mg}, 4.4 \mu \mathrm{mol})$ in a Schlenk flask $(10 \mathrm{~mL})$ was added a solution of 7 (2.7 mg, $9.6 \mu \mathrm{mol})$ in dioxane $(1 \mathrm{~mL})$. The resulting solution was stirred for $15 \mathrm{~min}$, and $\mathrm{KOH}\left(1.5 \mathrm{M} / \mathrm{H}_{2} \mathrm{O}, 100 \mu \mathrm{L}, 0.15 \mathrm{mmol}\right)$ was added. After stirring $15 \mathrm{~min}$, 4$\mathrm{MeOC}_{6} \mathrm{H}_{4} \mathrm{~B}(\mathrm{OH})_{2}(88 \mathrm{mg}, 0.58 \mathrm{mmol}$ ) was added followed by ethyl cinnamate (49 $\mu \mathrm{L}$, $0.29 \mathrm{mmol}$ ) and the reaction was stirred at $50{ }^{\circ} \mathrm{C}$ for $18 \mathrm{~h}$. Saturated $\mathrm{NH}_{4} \mathrm{Cl}$ was added, and the aqueous layer was extracted with $\mathrm{Et}_{2} \mathrm{O}(3 \times)$. The combined organic layers were washed with brine, dried over $\mathrm{MgSO}_{4}$, and the solvent was evaporated to give the crude. The desired product (59 mg, 72\%) was isolated by flash chromatography using 15\% $\mathrm{Et}_{2} \mathrm{O} /$ hexane as a colorless liquid. The enantioselectivity was 19\% ee (OD-H, $254 \mathrm{~nm}$, hexane:2-propanol $=95: 5$, flow rate $=1.0 \mathrm{~mL} / \mathrm{min}, \mathrm{t}_{\mathrm{r}(\text { minor) }}=7.13 \mathrm{~min}, \mathrm{t}_{\text {(major) }}=8.34$ $\min )$. IR (neat) $v=3030,2980,2835,1730,1510,1246,1031,699,667 \mathrm{~cm}^{-1} ;{ }^{1} \mathrm{H}$ NMR (300 MHz, $\left.\mathrm{CDCl}_{3}\right) \delta$ 7.31-7.15 (m, 7H), $6.83(\mathrm{~m}, 2 \mathrm{H}), 4.53(\mathrm{t}, 1 \mathrm{H}, J=8.1 \mathrm{~Hz}), 4.05$ (q, 2H, $J=7.2 \mathrm{~Hz}), 3.77$ (s, 3H), 3.04 (d, $2 \mathrm{H}, J=8.1 \mathrm{~Hz}), 1.13(\mathrm{t}, 3 \mathrm{H}, J=7.2 \mathrm{~Hz}) ;{ }^{13} \mathrm{C} \mathrm{NMR}$ 
(75.5 MHz, $\left.\mathrm{CDCl}_{3}\right) \delta 172.1,158.3,144.1,135.8,128.8,128.7,127.8,126.6,114.1,60.6$, 55.4, 46.5, 41.2, 14.3; HRMS-EI calcd for $\mathrm{C}_{18} \mathrm{H}_{20} \mathrm{O}_{3}[\mathrm{M}]^{+}$284.1412, found 284.1409.

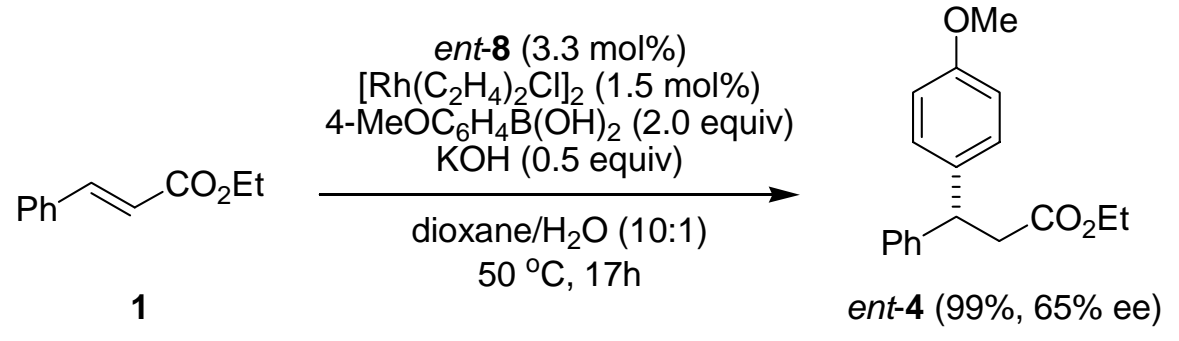

\section{(R)-Ethyl 3-(4-methoxyphenyl)-3-phenylpropanoate (ent-4).}

Following the general procedure in dioxane on $0.33 \mathrm{mmol}$ scale using ethyl cinnamate, 4methoxyphenylboronic acid (2.0 equiv), and ligand ent-8 with a reaction time of $17 \mathrm{~h}$, the desired product (93 mg, 99\%) was isolated by flash chromatography using 15\% $\mathrm{Et}_{2} \mathrm{O} /$ hexane as a colorless liquid. The enantioselectivity was $-65 \%$ ee (OD-H, $254 \mathrm{~nm}$, hexane:2-propanol $=95: 5$, flow rate $=1.0 \mathrm{~mL} / \mathrm{min}, \mathrm{t}_{\mathrm{r} \text { (major) }}=7.15 \mathrm{~min}, \mathrm{t}_{\mathrm{r} \text { (minor) }}=8.47$ $\min )$.
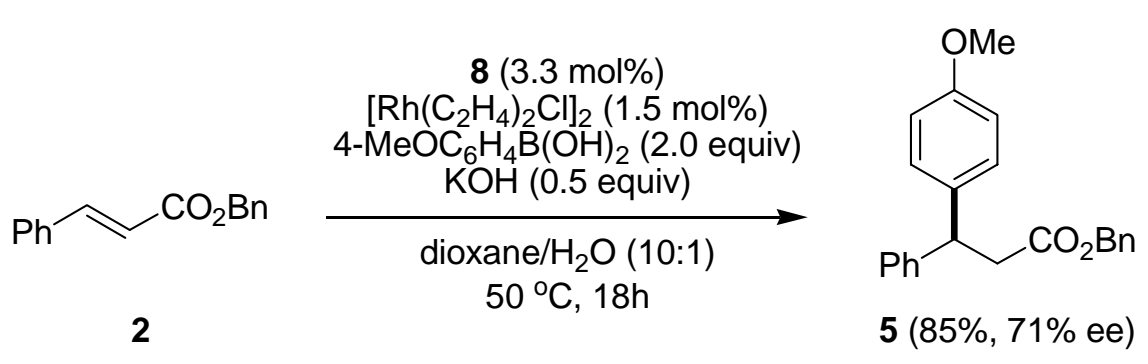

\section{(S)-Benzyl 3-(4-methoxyphenyl)-3-phenylpropanoate (5)}

Following the general procedure in dioxane on $0.31 \mathrm{mmol}$ scale using benzyl cinnamate, 4-methoxyphenylboronic acid (2.0 equiv), and ligand 8 with a reaction time of $18 \mathrm{~h}$, the desired product (91 mg, 85\%) was isolated by flash chromatography using 15\% $\mathrm{Et}_{2} \mathrm{O} /$ hexane as a colorless solid. The enantioselectivity was $71 \%$ ee (OD-H, $254 \mathrm{~nm}$, hexane:2-propanol $=90: 10$, flow rate $=1.0 \mathrm{~mL} / \mathrm{min}, \mathrm{t}_{\mathrm{r}(\operatorname{minor})}=9.18 \mathrm{~min}, \mathrm{t}_{\mathrm{r} \text { (major) }}=10.49$ 
$\min$ ). $\mathrm{mp}=79-80^{\circ} \mathrm{C}$; IR (neat) $v=3031,2960,2909,2837,1727,1246,1147,732,693$, $667 \mathrm{~cm}^{-1} ;{ }^{1} \mathrm{H}$ NMR (300 MHz, $\left.\mathrm{CDCl}_{3}\right) \delta$ 7.32-7.14 (m, 12H), 6.82 (m, 2H), 5.03 (s, 2H), 4.54 (t, 1H, $J=8.1 \mathrm{~Hz}$ ), 3.78 (s, 3H), 3.09 (d, 2H, $J=8.1 \mathrm{~Hz}) ;{ }^{13} \mathrm{C}$ NMR $(75.5 \mathrm{MHz}$, $\left.\mathrm{CDCl}_{3}\right) \delta 171.9,158.4,143.9,136.0,135.7,128.8,128.7,128.6,128.3,127.8,126.7$, 114.1, 66.5, 55.4, 46.5, 41.2; HRMS-EI calcd for $\mathrm{C}_{23} \mathrm{H}_{22} \mathrm{O}_{3}[\mathrm{M}]^{+}$346.1569, found 346.1564.
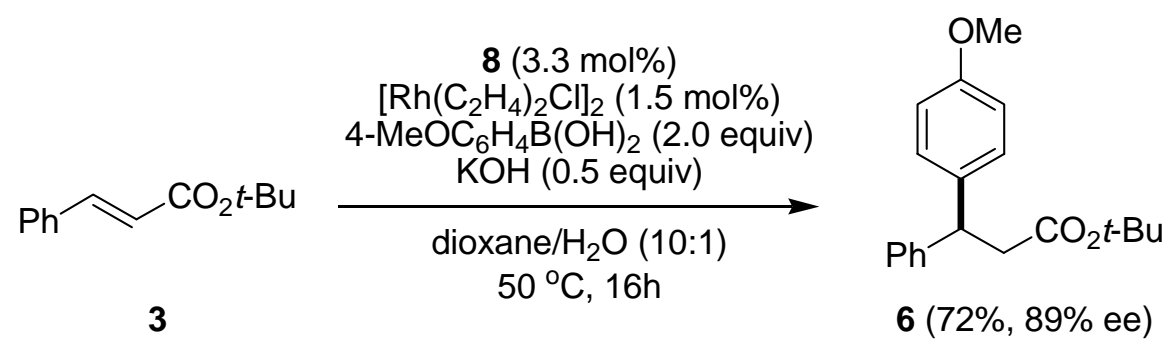

\section{(S)-tert-Butyl 3-(4-methoxyphenyl)-3-phenylpropanoate (6)}

Following the general procedure in dioxane on $0.29 \mathrm{mmol}$ scale using t-butyl cinnamate, 4-methoxyphenylboronic acid (2.0 equiv), and ligand 8 with a reaction time of $16 \mathrm{~h}$, the desired product (65 mg, 72\%) was isolated by flash chromatography using $10 \%$ $\mathrm{Et}_{2} \mathrm{O} /$ hexane as a colorless solid. The enantioselectivity was $89 \%$ ee (AD-H, $254 \mathrm{~nm}$, hexane:2-propanol $=98: 2$, flow rate $=0.35 \mathrm{~mL} / \mathrm{min}, \mathrm{t}_{\mathrm{r} \text { (major) }}=23.55 \mathrm{~min}, \mathrm{t}_{\mathrm{r} \text { (minor) }}=25.73$ $\min$ ). $\mathrm{mp}=70-71{ }^{\circ} \mathrm{C}$; IR (neat) $v=3030,3005,2969,2932,2838,1714,1512,1139$, 1029, $667 \mathrm{~cm}^{-1} ;{ }^{1} \mathrm{H}$ NMR (300 MHz, $\left.\mathrm{CDCl}_{3}\right) \delta$ 7.30-7.15 (m, 7H), $6.82(\mathrm{~m}, 2 \mathrm{H}), 4.44(\mathrm{t}$, 1H, $J=8.4 \mathrm{~Hz}$ ), 3.77 (s, 3H), 2.94 (d, 2H, $J=8.4 \mathrm{~Hz}), 1.29$ (s, 3H); ${ }^{13} \mathrm{C}$ NMR $(75.5$ $\left.\mathrm{MHz}, \mathrm{CDCl}_{3}\right) \delta 171.4,158.3,144.1,136.0,128.9,128.6,127.9,126.5,114.0,80.6,55.4$, 46.8, 42.5, 28.1; HRMS-EI calcd for $\mathrm{C}_{20} \mathrm{H}_{24} \mathrm{O}_{3}[\mathrm{M}]^{+}$312.1725, found 312.1722. 


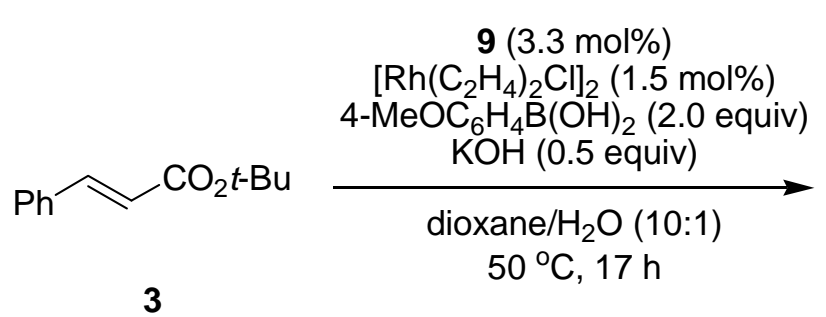

3

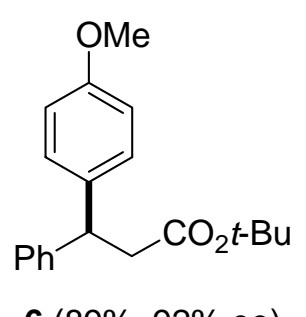

$6(89 \%, 92 \%$ ee)

\section{(S)-tert-Butyl 3-(4-methoxyphenyl)-3-phenylpropanoate (6)}

Following the general procedure in dioxane on $0.31 \mathrm{mmol}$ scale using t-butyl cinnamate, 4-methoxyphenylboronic acid (2.0 equiv), and ligand 9 with a reaction time of $17 \mathrm{~h}$, the desired product (86 mg, 89\%) was isolated by flash chromatography using $10 \%$ $\mathrm{Et}_{2} \mathrm{O} /$ hexane as a colorless solid. The enantioselectivity was $92 \%$ ee (AD-H, $254 \mathrm{~nm}$, hexane:2-propanol $=98: 2$, flow rate $=0.35 \mathrm{~mL} / \mathrm{min}, \mathrm{t}_{\mathrm{r} \text { (major) }}=24.29 \mathrm{~min}, \mathrm{t}_{\mathrm{r} \text { (minor) }}=26.56$ $\min )$.

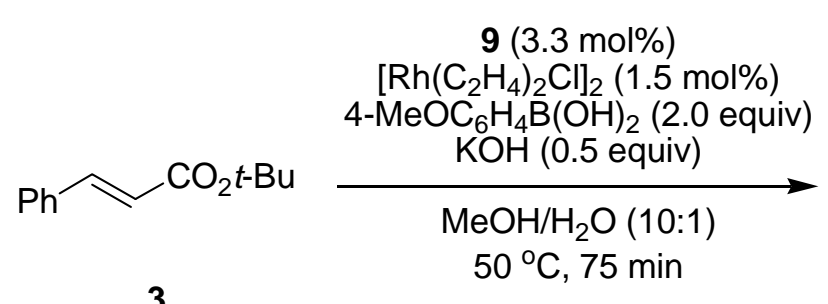

3

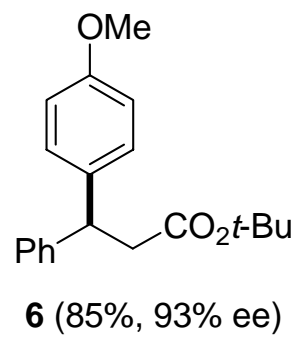

(S)-tert-Butyl 3-(4-methoxyphenyl)-3-phenylpropanoate (6). General Procedure in

\section{MeOH.}

To $\left[\mathrm{Rh}\left(\mathrm{C}_{2} \mathrm{H}_{4}\right) \mathrm{Cl}\right]_{2}(2.0 \mathrm{mg}, 5.1 \mu \mathrm{mol})$ in a Schlenk flask $(10 \mathrm{~mL})$ was added a solution of 9 (3.5 mg, $11.3 \mu \mathrm{mol})$ in $\mathrm{MeOH}(1 \mathrm{~mL})$. The resulting solution was stirred for $15 \mathrm{~min}$, and $\mathrm{KOH}\left(1.5 \mathrm{M} / \mathrm{H}_{2} \mathrm{O}, 113 \mu \mathrm{L}, 0.17 \mathrm{mmol}\right)$ was added. After stirring $15 \mathrm{~min}$, 4$\mathrm{MeOC}_{6} \mathrm{H}_{4} \mathrm{~B}(\mathrm{OH})_{2}$ (103 mg, $0.68 \mathrm{mmol}$ ) was added followed by $t$-butyl cinnamate (69 $\mu \mathrm{L}, 0.34 \mathrm{mmol}$ ) and the reaction was stirred at $50{ }^{\circ} \mathrm{C}$ for $75 \mathrm{~min}$. Saturated $\mathrm{NH}_{4} \mathrm{Cl}$ was added, and the aqueous layer was extracted with $\mathrm{Et}_{2} \mathrm{O}(3 \times)$. The combined organic layers 
were washed with brine, dried over $\mathrm{MgSO}_{4}$, and the solvent was evaporated to give the crude. The desired product (90 mg, 85\%) was isolated by flash chromatography using $15 \% \mathrm{Et}_{2} \mathrm{O} /$ hexane as a colorless liquid. The enantioselectivity was 93\% ee (AD-H, 254 $\mathrm{nm}$, hexane:2-propanol $=98: 2$, flow rate $=0.35 \mathrm{~mL} / \mathrm{min}, \mathrm{t}_{\mathrm{r} \text { (major) }}=24.25 \mathrm{~min}, \mathrm{t}_{\mathrm{r} \text { (minor) }}=$ $26.63 \mathrm{~min}) \cdot[\alpha]_{\mathrm{D}}^{26}+1.6($ c $0.96, \mathrm{MeOH})$.

\section{1,4-Addition to t-Butyl Cinnamates Derivatives}

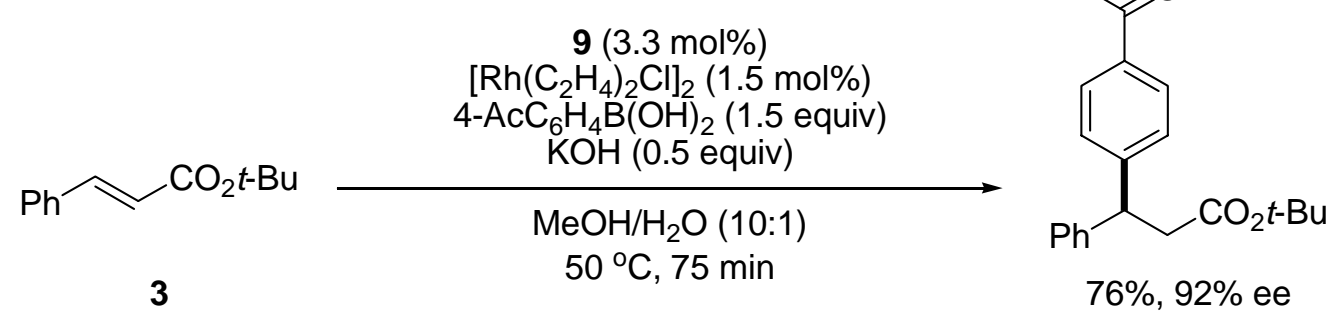

\section{(S)-tert-Butyl 3-(4-acetylphenyl)-3-phenylpropanoate}

Following the general procedure in $\mathrm{MeOH}$ on $0.3 \mathrm{mmol}$ scale using $t$-butyl cinnamate, 4acetylphenylboronic acid (1.5 equiv), and ligand 9 with a reaction time of $75 \mathrm{~min}$, the desired product (74 mg, 76\%) was isolated by flash chromatography using hexanes/EtOAc 7:1 as a colorless solid. The enantioselectivity was 92\% ee (AD-H, 254 $\mathrm{nm}$, hexane:2-propanol $=98: 2$, flow rate $=1.0 \mathrm{~mL} / \mathrm{min}, \mathrm{t}_{\mathrm{r}(\text { major })}=19.7 \mathrm{~min}, \mathrm{t}_{\mathrm{r}(\operatorname{minor})}=21.5$ $\min ) .[\alpha]_{\mathrm{D}}^{34}+7.5\left(c\right.$ 1.03, $\mathrm{CHCl}_{3}$ ). IR (neat) $v=3028,3005,2979,2931,1720,1680$, 1597, 1495, 1365, 1261, 1145, 1116, 957, $850 \mathrm{~cm}^{-1} ;{ }^{1} \mathrm{H}$ NMR (300 MHz, $\left.\mathrm{CDCl}_{3}\right) \delta 7.88$ (d, 2H, $J=8.4 \mathrm{~Hz}$ ), 7.35 (d, 2H, $J=8.4 \mathrm{~Hz}$ ), 7.32-7.17 (m, 5H), 4.54 (t, $1 \mathrm{H}, J=8.1 \mathrm{~Hz}$ ), $2.98(\mathrm{~d}, 2 \mathrm{H}, J=8.1 \mathrm{~Hz}), 2.56$ (s, 3H), $1.28(\mathrm{~s}, 9 \mathrm{H}) ;{ }^{13} \mathrm{C} \mathrm{NMR}\left(75.5 \mathrm{MHz}, \mathrm{CDCl}_{3}\right) \delta$ 197.5, 170.5, 149.0, 142.6, 135.4, 128.6, 127.9, 127.6, 126.7, 80.8, 47.4, 41.7, 28.0, 26.7; HRMS-EI calcd for $\mathrm{C}_{21} \mathrm{H}_{24} \mathrm{O}_{3}[\mathrm{M}]^{+}$324.1725, found 324.1759. 


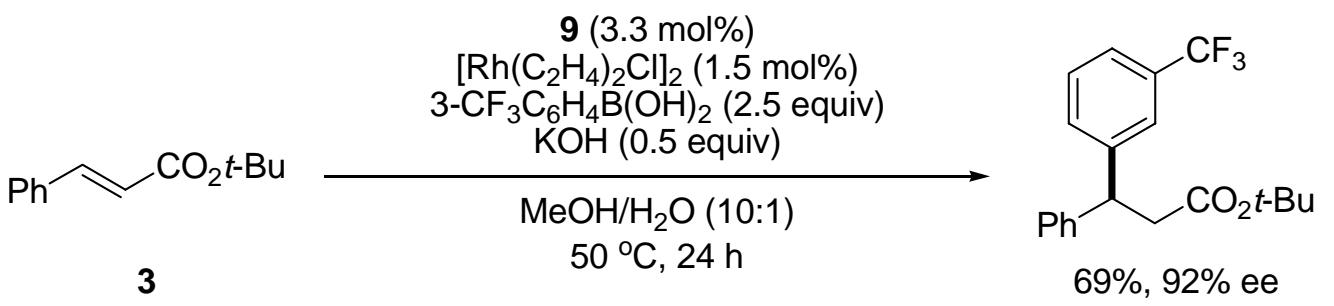

\section{(S)-tert-Butyl 3-(3-trifluoromethylphenyl)-3-phenylpropanoate}

Following the general procedure in $\mathrm{MeOH}$ on $0.39 \mathrm{mmol}$ scale using $t$-butyl cinnamate, 3-trifluoromethylphenylboronic acid (2.5 equiv), and ligand 9 with a reaction time of 24 $\mathrm{h}$, the desired product (94 mg, 69\%) was isolated by flash chromatography using $5 \%$ EtOAc/hexane as a colorless oil. The enantioselectivity was 92\% ee (AD-H, $254 \mathrm{~nm}$, hexane:2-propanol $=98: 2$, flow rate $=1.0 \mathrm{~mL} / \mathrm{min}, \mathrm{t}_{\mathrm{r}}$ (major) $=4.44 \mathrm{~min}, \mathrm{t}_{\mathrm{r} \text { (minor) }}=5.69$ $\min ) .[\alpha]_{\mathrm{D}}^{29}-5.4$ (c 0.8, MeOH). IR (neat) $v=3066,3030,2979,2933,1706,1444$, 1366, 1327, 1160, 1119, $1075 \mathrm{~cm}^{-1} ;{ }^{1} \mathrm{H}$ NMR (300 MHz, $\left.\mathrm{CDCl}_{3}\right) \delta 7.53$ (bs, $\left.1 \mathrm{H}\right) .7 .47-$ 7.35 (m, 3 H), 7.32-7.18 (m, 5 H), 4.55 (t, 1 H, $J=8.1$ Hz), 2.98 (d, 2 H, $J=8.4$ Hz), 1.28 (s, $9 \mathrm{H}) ;{ }^{13} \mathrm{C}$ NMR $\left(75.5 \mathrm{MHz}, \mathrm{CDCl}_{3}\right) \delta 170.6,144.6,142.7,131.4\left(\mathrm{q},{ }^{4} J_{C F}=1.1\right.$ $\mathrm{Hz}), 130.8\left(\mathrm{q},{ }^{2} J_{C F}=31.9 \mathrm{~Hz}\right), 128.9,128.7,127.7,126.8,124.4\left(\mathrm{q},{ }^{3} J_{C F}=3.8 \mathrm{~Hz}\right), 124.1$ $\left(\mathrm{q},{ }^{1} J_{C F}=272 \mathrm{~Hz}\right), 123.4\left(\mathrm{q},{ }^{3} J_{C F}=4.0 \mathrm{~Hz}\right)$, ; HRMS-EI calcd for $\mathrm{C}_{20} \mathrm{H}_{21} \mathrm{~F}_{3} \mathrm{O}_{2}[\mathrm{M}]^{+}$ 350.1489, found 350.1483.

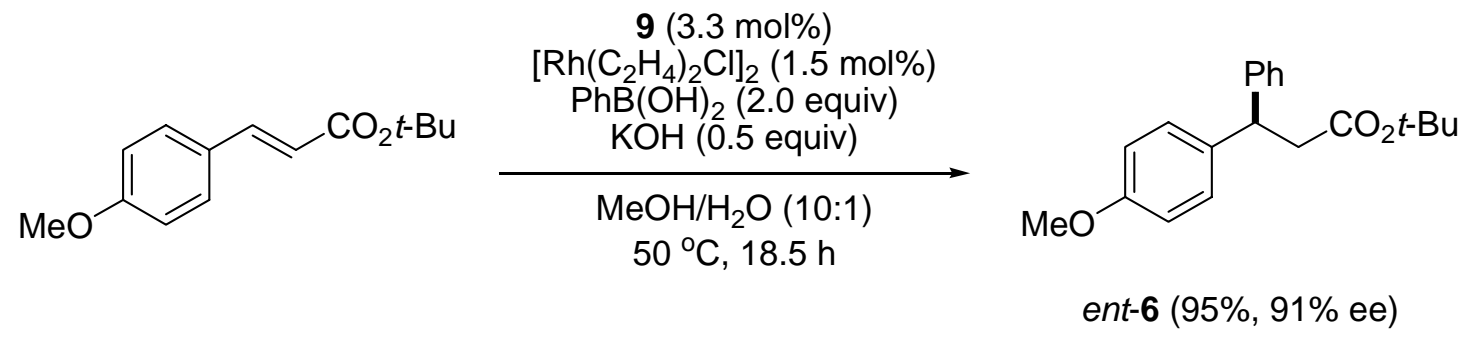

\section{(R)-tert-Butyl 3-(4-methoxyphenyl)-3-phenylpropanoate (ent-6)}

Following the general procedure in $\mathrm{MeOH}$ on 0.39 mmol scale using t-butyl 4methoxycinnamate, phenylboronic acid (2.0 equiv), and ligand $\mathbf{9}$ with a reaction time of 
$18.5 \mathrm{~h}$, the desired product (115 $\mathrm{mg}, 95 \%$ ) was isolated by flash chromatography using 10\% EtOAc/hexane as a light yellow oil. The enantioselectivity was 91\% ee (AD-H, 254 $\mathrm{nm}$, hexane:2-propanol $=98: 2$, flow rate $=0.35 \mathrm{~mL} / \mathrm{min}, \mathrm{t}_{\mathrm{r} \text { (minor) }}=24.16 \mathrm{~min}, \mathrm{t}_{\mathrm{r} \text { (major) }}=$ $26.14 \mathrm{~min}) .[\alpha]_{\mathrm{D}}{ }^{31}-0.92$ (c $\left.0.86, \mathrm{MeOH}\right)$. The spectral data were identical to 6.
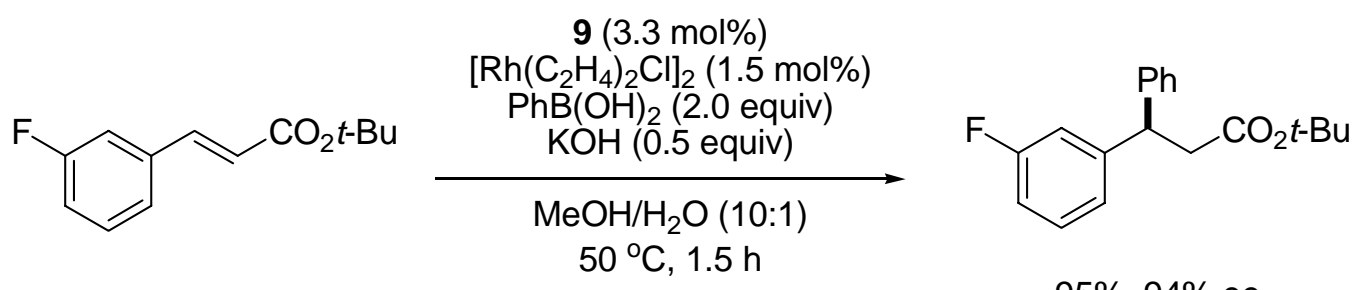

$95 \%, 94 \%$ ee

\section{(R)-tert-Butyl 3-(3-fluorophenyl)-3-phenylpropanoate}

Following the general procedure in $\mathrm{MeOH}$ on $0.39 \mathrm{mmol}$ scale using t-butyl 3fluorocinnamate, phenylboronic acid (2.0 equiv), and ligand $\mathbf{9}$ with a reaction time of 1.5 h, the desired product (111 mg, 95\%) was isolated by flash chromatography using 5\% EtOAc/hexane as a colorless oil. The enantioselectivity was 94\% ee (OJ-H, $254 \mathrm{~nm}$, hexane:2-propanol $=99: 1$, flow rate $=0.50 \mathrm{~mL} / \mathrm{min}, \mathrm{t}_{\mathrm{r} \text { (minor) }}=32.37 \mathrm{~min}, \mathrm{t}_{\mathrm{r} \text { (major) }}=34.37$ $\min ) .[\alpha]_{D}{ }^{25}-7.1$ (c 1.2, $\left.\mathrm{C}_{6} \mathrm{H}_{6}\right)$; IR (neat) $v=3066,3030,2977,2929,1725,1366,1247$, 1141, $699 \mathrm{~cm}^{-1} ;{ }^{1} \mathrm{H}$ NMR (300 MHz, $\mathrm{CDCl}_{3}$ ) $\delta$ 7.33-7.18 (m, 6H), 7.04 (br d, $1 \mathrm{H}, J=7.5$ Hz), 6.96 (dt, 1H, $J=10.2,2.4 \mathrm{~Hz}$ ), 6.88 (tdd, 1H, $J=8.7,2.4,0.9 \mathrm{~Hz}), 4.49$ (t, $1 \mathrm{H}, J=$ $8.4 \mathrm{~Hz}$ ), 2.96 (d, 2H, $J=8.4 \mathrm{~Hz}), 1.30$ (s, 9H); $\left.{ }^{13} \mathrm{C} \mathrm{NMR} \mathrm{(75.5} \mathrm{MHz,} \mathrm{CDCl}_{3}\right) \delta$ 170.6, $162.7\left(\mathrm{~d},{ }^{1} J_{\mathrm{C}-\mathrm{F}}=244.8 \mathrm{~Hz}\right), 146.1\left(\mathrm{~d},{ }^{3} J_{\mathrm{C}-\mathrm{F}}=6.7 \mathrm{~Hz}\right), 142.8,129.8\left(\mathrm{~d},{ }^{3} J_{\mathrm{C}-\mathrm{F}}=8.5 \mathrm{~Hz}\right)$, 128.5, 127.6, 126.6, $123.3\left(\mathrm{~d},{ }^{4} J_{\mathrm{C}-\mathrm{F}}=2.4 \mathrm{~Hz}\right), 114.6\left(\mathrm{~d},{ }^{2} J_{\mathrm{C}-\mathrm{F}}=21.4 \mathrm{~Hz}\right), 113.3\left(\mathrm{~d},{ }^{2} J_{\mathrm{C}-\mathrm{F}}=\right.$ $21.4 \mathrm{~Hz}$ ), 80.7, 47.2, 41.9, 27.9; HRMS-EI calcd for $\mathrm{C}_{19} \mathrm{H}_{21} \mathrm{FO}_{2}[\mathrm{M}]^{+}$300.1526, found 300.1522. 

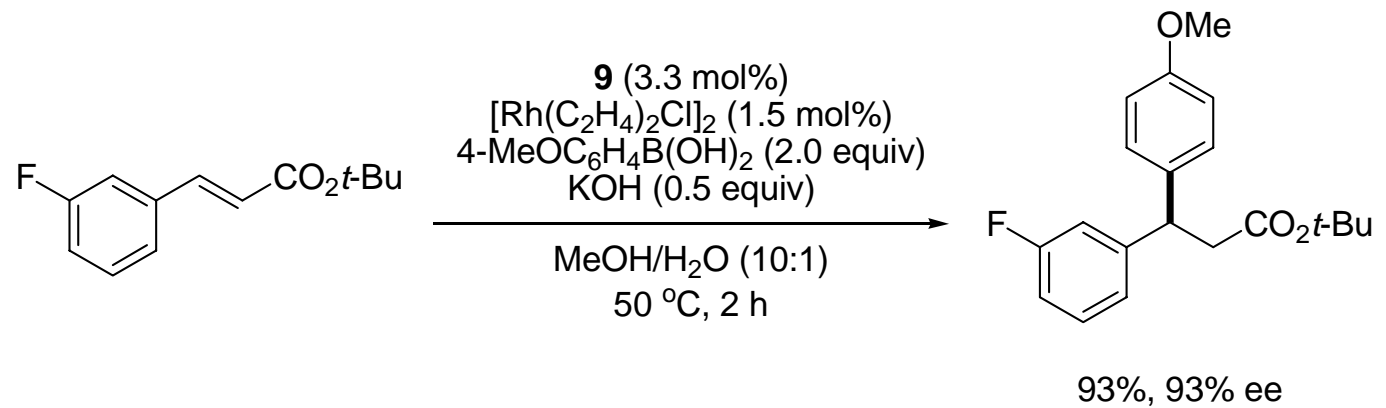

\section{(R)-tert-Butyl 3-(3-fluorophenyl)-3-(4-methoxyphenyl)propanoate}

Following the general procedure in $\mathrm{MeOH}$ on $0.39 \mathrm{mmol}$ scale using t-butyl 3fluorocinnamate, 4-methoxyphenylboronic acid (2.0 equiv), and ligand 9 with a reaction time of $2 \mathrm{~h}$, the desired product (120 mg, 93\%) was isolated by flash chromatography using 5\% EtOAc/hexane as a colorless oil. The enantioselectivity was 93\% ee (OJ-H, 254 $\mathrm{nm}$, hexane:2-propanol $=99: 1$, flow rate $=0.50 \mathrm{~mL} / \mathrm{min}, \mathrm{t}_{\mathrm{r} \text { (minor) }}=28.57 \mathrm{~min}, \mathrm{t}_{\mathrm{r} \text { (major) }}=$ $33.79 \mathrm{~min}) .[\alpha]_{\mathrm{D}}{ }^{28}-2.4\left(c 0.84, \mathrm{C}_{6} \mathrm{H}_{6}\right)$; $\mathrm{mp}=67-68^{\circ} \mathrm{C}$; IR (neat) $v=3064,2982,2934$, 2838, 1711, 1512, 1247, 1139, 1026, $687 \mathrm{~cm}^{-1} ;{ }^{1} \mathrm{H}$ NMR (300 MHz, $\left.\mathrm{CDCl}_{3}\right) \delta$ 7.27-7.12 (m, 3H), 7.03-6.80 (m, 5H), 4.43 (t, 1H, $J=8.1 \mathrm{~Hz}$ ), 3.77 (S, 3H), 2.91 (d, 2H, $J=8.1$ $\mathrm{Hz}), 1.30$ (s, 9H); ${ }^{13} \mathrm{C}$ NMR $\left(75.5 \mathrm{MHz}, \mathrm{CDCl}_{3}\right) \delta 170.7,162.7\left(\mathrm{~d},{ }^{1} J_{\mathrm{C}-\mathrm{F}}=245.4 \mathrm{~Hz}\right)$, 158.1, $146.5\left(\mathrm{~d},{ }^{3} J_{\mathrm{C}-\mathrm{F}}=6.7 \mathrm{~Hz}\right), 134.9,129.7\left(\mathrm{~d},{ }^{3} J_{\mathrm{C}-\mathrm{F}}=7.9 \mathrm{~Hz}\right), 128.5,123.2\left(\mathrm{~d},{ }^{4} J_{\mathrm{C}-\mathrm{F}}=\right.$ $2.5 \mathrm{~Hz}), 114.5\left(\mathrm{~d},{ }^{2} J_{\mathrm{C}-\mathrm{F}}=21.4 \mathrm{~Hz}\right), 113.8,113.1\left(\mathrm{~d},{ }^{2} J_{\mathrm{C}-\mathrm{F}}=21.4 \mathrm{~Hz}\right), 80.6,55.2,46.4$, 42.1, 27.9; HRMS-EI calcd for $\mathrm{C}_{20} \mathrm{H}_{23} \mathrm{FO}_{3}[\mathrm{M}]^{+}$330.1631, found 330.1627. 


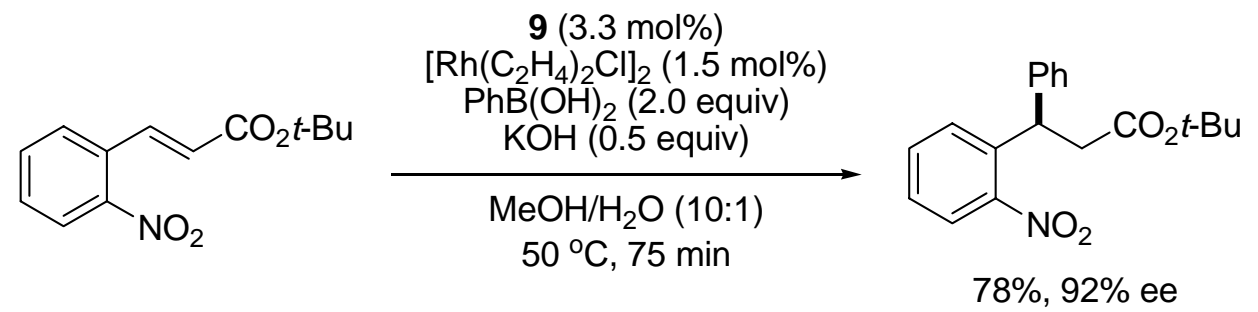

\section{(R)-tert-Butyl 3-(2-nitrophenyl)-3-phenylpropanoate}

Following the general procedure in $\mathrm{MeOH}$ on 0.40 mmol scale using t-butyl 2nitrocinnamate, phenylboronic acid (2.0 equiv), and ligand 9 with a reaction time of 75 min, the desired product (102 mg, 78\%) was isolated by flash chromatography using 10\% EtOAc/hexane as a colorless oil. The enantioselectivity was 92\% ee (AD-H, $254 \mathrm{~nm}$, hexane:2-propanol $=99: 1$, flow rate $=0.50 \mathrm{~mL} / \mathrm{min}, \mathrm{t}_{\mathrm{r}(\text { minor })}=29.43 \mathrm{~min}, \mathrm{t}_{\mathrm{r}(\text { major })}=39.13$ $\min ) \cdot[\alpha]_{\mathrm{D}}{ }^{27}+13.3\left(c 0.42, \mathrm{CHCl}_{3}\right)$. IR (neat) $v=33066,3026,2971,1725,1524,1354$, 1256, $1148 \mathrm{~cm}^{-1}$; ${ }^{1} \mathrm{H}$ NMR (300 MHz, $\mathrm{CDCl}_{3}$ ) $\delta 7.77$ (dd, $1 \mathrm{H}, J=8.1,1.2 \mathrm{~Hz}$ ), 7.56-7.44 (m, 2 H), 7.36-7.17 (m, 6 H), 5.15 (t, 1 H, $J=8.4$ Hz), 3.07-2.93 (m, 2 H), 1.26 (s, 9 H); ${ }^{13} \mathrm{C}$ NMR $\left(75.5 \mathrm{MHz}, \mathrm{CDCl}_{3}\right) \delta 170.0,149.8,141.6,137.5,132.5,129.3,128.6,127.8$, 127.3, 126.9, 124.4, 80.9, 42.0, 41.1, 27.7; HRMS-EI calcd for $\mathrm{C}_{15} \mathrm{H}_{12} \mathrm{NO}_{3}\left[\mathrm{M}-\mathrm{OC}_{4} \mathrm{H}_{9}\right]^{+}$ 254.0817, found 254.0813.
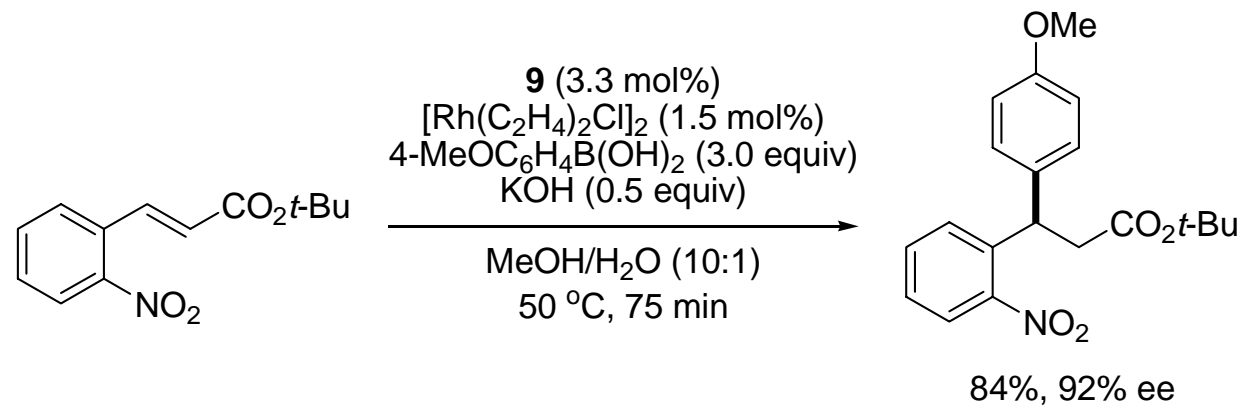

\section{(R)-tert-Butyl 3-(4-methoxyphenyl)-3-(2-nitrophenyl)propanoate}

Following the general procedure in $\mathrm{MeOH}$ on $0.39 \mathrm{mmol}$ scale using t-butyl 2nitrocinnamate, 4-methoxyphenylboronic acid (3.0 equiv), and ligand 9 with a reaction 
time of $75 \mathrm{~min}$, the desired product (117 $\mathrm{mg}, 84 \%$ ) was isolated by flash chromatography using 10\% EtOAc/hexane as a light yellow oil. The enantioselectivity was 92\% ee (AD$\mathrm{H}, 254 \mathrm{~nm}$, hexane:2-propanol $=98: 2$, flow rate $=0.5 \mathrm{~mL} / \mathrm{min}, \mathrm{t}_{\mathrm{r} \text { (minor) }}=39.29 \mathrm{~min}, \mathrm{t}_{\mathrm{r}}$ $($ major) $=53.89 \mathrm{~min}) .[\alpha]_{\mathrm{D}}^{29}+35.1(c 0.94, \mathrm{MeOH})$. IR (neat) $v=2977,1728,1610,1527$, 1512, 1355, 1252, 1151, $1034 \mathrm{~cm}^{-1} ;{ }^{1} \mathrm{H}$ NMR (300 MHz, $\left.\mathrm{CDCl}_{3}\right) \delta$ 7.79-7.76 (m, $1 \mathrm{H}$ ), 7.56-7.44 (m, 2 H), 7.37-7.31 (m, 1 H), 7.21-7.17 (m, 2 H), 6.86-6.81 (m, 2 H), 5.10 (t, 1 H, $J=8.1 \mathrm{~Hz}$ ), 3.77 (s, $3 \mathrm{H}$ ), 3.05-2.90 (m, $2 \mathrm{H}), 1.28$ (s, $9 \mathrm{H}) ;{ }^{13} \mathrm{C}$ NMR (75.5 MHz, $\left.\mathrm{CDCl}_{3}\right) \delta 170.1,158.4,149.8,137.9,133.7,132.5,129.2,128.8,127.2,124.4,113.9$, 80.9, 55.2, 42.2, 40.4, 27.7; HRMS-EI calcd for $\mathrm{C}_{16} \mathrm{H}_{15} \mathrm{NO}_{5}\left[\mathrm{M}-\mathrm{C}_{4} \mathrm{H}_{8}\right]^{+}$301.0945, found 301.0946.

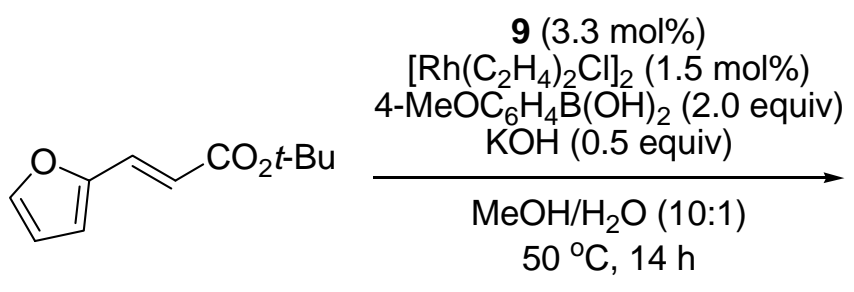

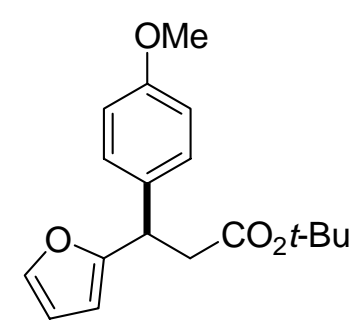

$62 \%, 92 \%$ ee

\section{(R)-tert-Butyl 3-(furan-2-yl)-3-(4-methoxyphenyl)propanoate}

Following the general procedure in $\mathrm{MeOH}$ on $0.37 \mathrm{mmol}$ scale using $t$-butyl 3-(furan-2yl)acrylate, 4-methoxyphenylboronic acid (2.0 equiv), and ligand 9 with a reaction time of $14 \mathrm{~h}$, the desired product (68 $\mathrm{mg}, 62 \%$ ) was isolated by flash chromatography using 10\% EtOAc/hexane as a colorless oil. The enantioselectivity was 92\% ee (AD-H, 254 $\mathrm{nm}$, hexane:2-propanol $=99: 1$, flow rate $=0.50 \mathrm{~mL} / \mathrm{min}, \mathrm{t}_{\mathrm{r} \text { (major) }}=18.17 \mathrm{~min}, \mathrm{t}_{\mathrm{r} \text { (minor) }}=$ $23.64 \mathrm{~min}) .[\alpha]_{\mathrm{D}}^{27}-38.2$ (c 0.66, $\mathrm{CHCl}_{3}$ ). IR (neat) $v=2976,2932,2836,1727,1511$, 1246, 1145, $730 \mathrm{~cm}^{-1} ;{ }^{1} \mathrm{H}$ NMR (300 MHz, $\left.\mathrm{CDCl}_{3}\right) \delta 7.30$ (dd, $\left.1 \mathrm{H}, J=1.8,0.6 \mathrm{~Hz}\right), 7.18$ 
(m, 2H), $6.84(\mathrm{~m}, 2 \mathrm{H}), 6.27$ (dd, 1H, $J=3.3,1.8 \mathrm{~Hz}), 6.02(\mathrm{dt}, 1 \mathrm{H}, J=3.3,0.6 \mathrm{~Hz}), 4.44$ (t, $1 \mathrm{H}, J=8.1 \mathrm{~Hz}$ ), 3.78 (s, 3H), 2.98 (dd, 1H, $J=15.0,8.1 \mathrm{~Hz}$ ), 2.77 (dd, 1H, $J=15.0$, 8.1 Hz), 1.33 (s, 9H); ${ }^{13} \mathrm{C}$ NMR (75.5 MHz, $\left.\mathrm{CDCl}_{3}\right) \delta$ 170.9. 158.7. 157.0. 141.7. 133.5. 129.0. 114.0. 110.2. 105.6. 80.8. 55.4. 41.3. 41.1. 28.1; HRMS-EI calcd for $\mathrm{C}_{18} \mathrm{H}_{22} \mathrm{O}_{4}$ $[\mathrm{M}]^{+}$302.1518, found 302.1514.
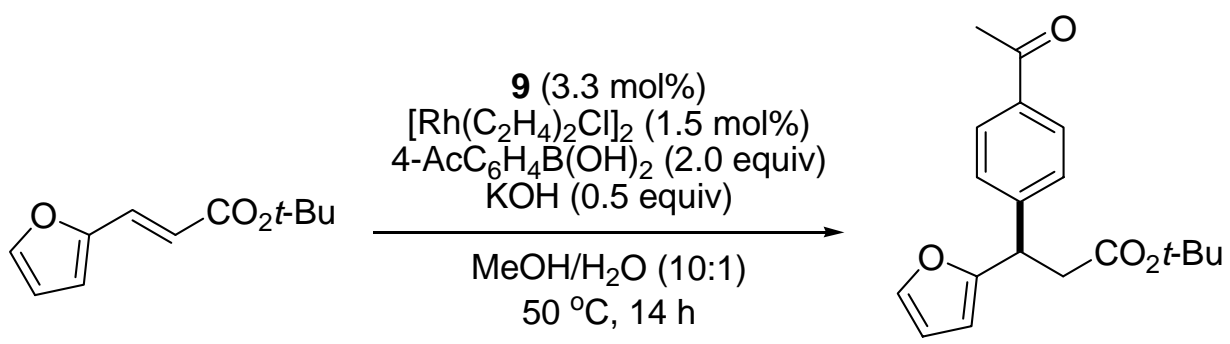

$68 \%, 91 \%$ ee

\section{(R)-tert-Butyl 3-(4-acetylphenyl)-3-(furan-2-yl)propanoate}

Following the general procedure in $\mathrm{MeOH}$ on $0.39 \mathrm{mmol}$ scale using $t$-butyl 3-(furan-2yl)acrylate, 4-acetylphenylboronic acid (2.0 equiv), and ligand 9 with a reaction time of $14 \mathrm{~h}$, the desired product ( $82 \mathrm{mg}, 68 \%$ ) was isolated by flash chromatography using $10 \%$ then $20 \% \mathrm{EtOAc/hexane} \mathrm{as} \mathrm{a} \mathrm{colorless} \mathrm{oil.} \mathrm{The} \mathrm{enantioselectivity} \mathrm{was} 91 \%$ ee (AD-H, $254 \mathrm{~nm}$, hexane:2-propanol $=98: 2$, flow rate $=1.0 \mathrm{~mL} / \mathrm{min}, \mathrm{t}_{\mathrm{r} \text { (major) }}=15.58 \mathrm{~min}, \mathrm{t}_{\mathrm{r} \text { (minor) }}$ $=20.35 \mathrm{~min}) \cdot[\alpha]_{\mathrm{D}}^{30}-41.3\left(c 0.8, \mathrm{CHCl}_{3}\right)$. IR (neat) $v=3006,2977,2933,1726,1682$, 1265, 1146, $734 \mathrm{~cm}^{-1} ;{ }^{1} \mathrm{H}$ NMR (300 MHz, $\mathrm{CDCl}_{3}$ ) $\delta 7.92$ (m, 2H), 7.35 (m, 2H), 7.31 (dd, $1 \mathrm{H}, J=1.8,0.9 \mathrm{~Hz}), 6.28$ (dd, $1 \mathrm{H}, J=3.3,1.8 \mathrm{~Hz}), 6.06$ (dt, $1 \mathrm{H}, J=3.3,0.9 \mathrm{~Hz}$ ), 4.55 (br t, 1H, $J=8.1 \mathrm{~Hz}$ ), 3.01 (dd, 1H, $J=15.3,7.5 \mathrm{~Hz}$ ), 2.83 (dd, $1 \mathrm{H}, J=15.3,8.3$ Hz), 2.57 (s, 3H), 1.32 (s, 9H); ${ }^{13} \mathrm{C}$ NMR (75.5 MHz, $\left.\mathrm{CDCl}_{3}\right) \delta 197.5,170.0,155.3$, 146.5, 141.7, 135.8, 128.5, 128.0, 110.1, 105.9, 80.9, 41.6, 40.5, 28.0, 26.7; HRMS-EI calcd for $\mathrm{C}_{19} \mathrm{H}_{22} \mathrm{O}_{4}\left[\mathrm{M}-\mathrm{C}_{4} \mathrm{H}_{9}\right]^{+}$257.0814, found 257.0806. 

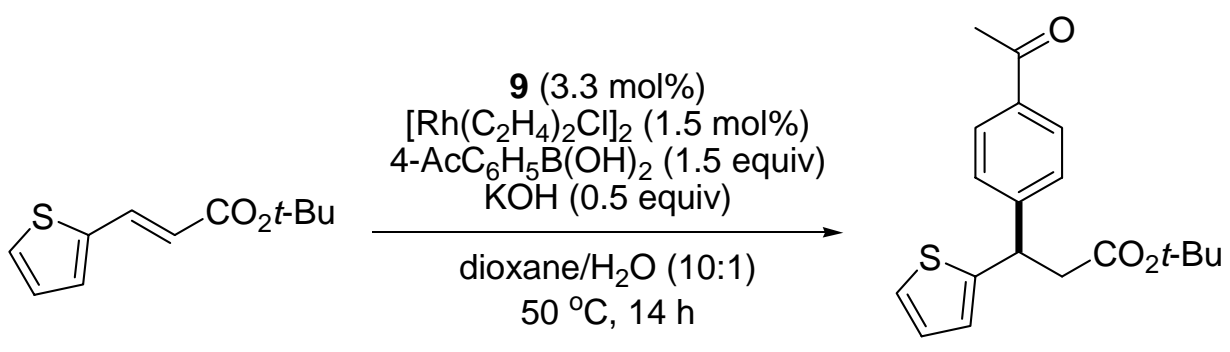

65\%, 91\% ee

\section{(R)-tert-Butyl 3-(4-acetylphenyl)-3-(thiophen-2-yl)propanoate}

Following the general procedure in dioxane on $0.39 \mathrm{mmol}$ scale using tert-butyl-3(thiophen-2-yl)acrylate, 4-acetylphenylboronic acid (1.5 equiv), and ligand 9 with a reaction time of $14 \mathrm{~h}$, the desired product (64 mg, 65\%) was isolated by flash chromatography using hexanes/EtOAc 10:1 as a colorless oil. The enantioselectivity was 91\% ee $\left(\mathrm{OD}-\mathrm{H}, 254 \mathrm{~nm}\right.$, hexane:2-propanol $=96: 4$, flow rate $=0.5 \mathrm{~mL} / \mathrm{min}, \mathrm{t}_{\mathrm{r}(\operatorname{minor})}=$ 18.9 min, $\left.\mathrm{t}_{\mathrm{r}(\mathrm{major})}=20.1 \mathrm{~min}\right) .[\alpha]_{\mathrm{D}}^{29}-14.5\left(\mathrm{c} 0.95, \mathrm{CHCl}_{3}\right)$. IR (neat) $v=2976,1727$, 1686, 1606, 1366, 1267, 1147, 956, $843 \mathrm{~cm}^{-1} ;{ }^{1} \mathrm{H}$ NMR $\left(300 \mathrm{MHz}, \mathrm{CDCl}_{3}\right) \delta 7.90$ (d, 2H, $J=8.1 \mathrm{~Hz}), 7.38(\mathrm{~d}, 2 \mathrm{H}, J=8.1 \mathrm{~Hz}), 7.17-7.14(\mathrm{~m}, 1 \mathrm{H}), 6.91(\mathrm{~m}, 1 \mathrm{H}), 6.83(\mathrm{~m}, 1 \mathrm{H})$, 4.76 (t, $1 \mathrm{H}, J=8.1 \mathrm{~Hz}$ ), 3.01 (dd, $1 \mathrm{H}, J=15.3,7.5 \mathrm{~Hz}), 2.83(\mathrm{dd}, 1 \mathrm{H}, J=15.3,8.3 \mathrm{~Hz}$ ), 2.57 (s, 3H), 1.30 (s, 9H); ${ }^{13} \mathrm{C}$ NMR $\left(75.5 \mathrm{MHz}, \mathrm{CDCl}_{3}\right) \delta$ 197.5, 169.9, 148.4, 146.4, 135.7, 128.6, 127.8, 126.6, 124.2, 124.1, 81.6, 43.0, 42.9, 28.0, 26.7; HRMS-EI calcd for $\mathrm{C}_{19} \mathrm{H}_{22} \mathrm{O}_{3} \mathrm{~S}\left[\mathrm{M}-\mathrm{C}_{4} \mathrm{H}_{8}\right]^{+}$274.0659, found 274.0659. 


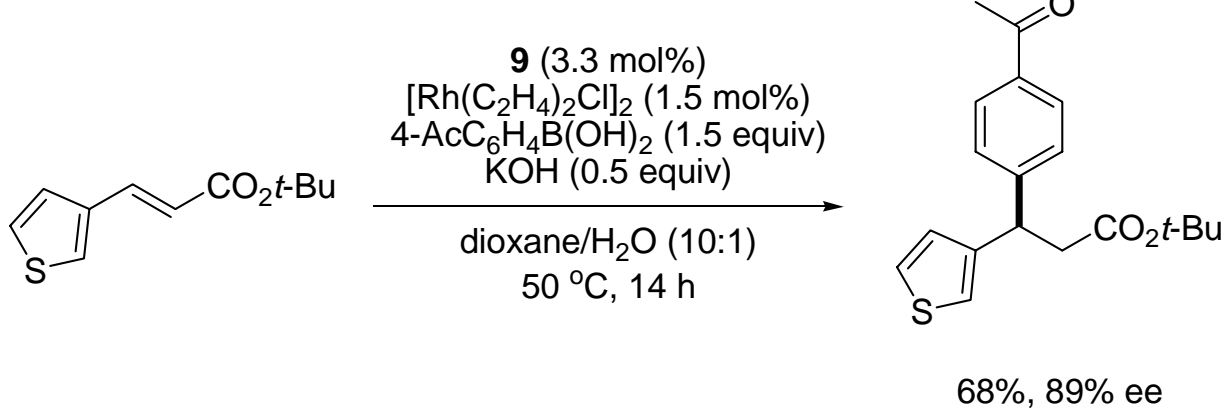

\section{(R)-tert-Butyl 3-(4-acetylphenyl)-3-(thiophen-3-yl)propanoate}

Following the general procedure in dioxane on $0.30 \mathrm{mmol}$ scale using tert-butyl 3(thiophen-3-yl)acrylate, 4-acetylphenylboronic acid (1.5 equiv), and ligand 9 with a reaction time of $14 \mathrm{~h}$, the desired product (67 $\mathrm{mg}, 68 \%$ ) was isolated by flash chromatography using hexane/EtOAc (3:1) as a colorless oil. The enantioselectivity was 89\% ee $\left(\mathrm{AD}-\mathrm{H}, 254 \mathrm{~nm}\right.$, hexane:2-propanol $=98: 2$, flow rate $=1.0 \mathrm{~mL} / \mathrm{min}, \mathrm{t}_{\mathrm{r}}$ (major) $=$ 22.4 min, $\left.\mathrm{t}_{\mathrm{r} \text { (minor) }}=25.4 \mathrm{~min}\right) \cdot[\alpha]_{\mathrm{D}}{ }^{34}-37.2$ (c 1.0, $\left.\mathrm{CHCl}_{3}\right)$. IR (neat) $v=3103,2976$, 2929, 1723, 1681, 1606, 1366, 1357, 1265, 1141, 956, 830, $779 \mathrm{~cm}^{-1} ;{ }^{1} \mathrm{H}$ NMR (300 MHz, $\left.\mathrm{CDCl}_{3}\right) \delta 7.89(\mathrm{~d}, 2 \mathrm{H}, J=8.4 \mathrm{~Hz}), 7.33(\mathrm{~d}, 2 \mathrm{H}, J=8.4 \mathrm{~Hz}), 7.27(\mathrm{~m}, 1 \mathrm{H}), 7.01(\mathrm{~m}$, 1H), $6.88(\mathrm{~m}, 1 \mathrm{H}), 4.59$ (t, 1H, $J=8.1 \mathrm{~Hz}), 3.04-2.86(\mathrm{~m}, 2 \mathrm{H}), 2.58$ (s, 3H), 1.30 (s, 9H); ${ }^{13} \mathrm{C}$ NMR $\left(75.5 \mathrm{MHz}, \mathrm{CDCl}_{3}\right) \delta 197.5,170.4,148.7,143.3,135.5,128.6,127.9,127.3$, 125.9, 120.6, 80.9, 43.2, 42.2, 28.0, 26.7; HRMS-EI calcd for $\mathrm{C}_{19} \mathrm{H}_{22} \mathrm{SO}_{3}[\mathrm{M}]^{+}$330.1290, found 330.1292 . 


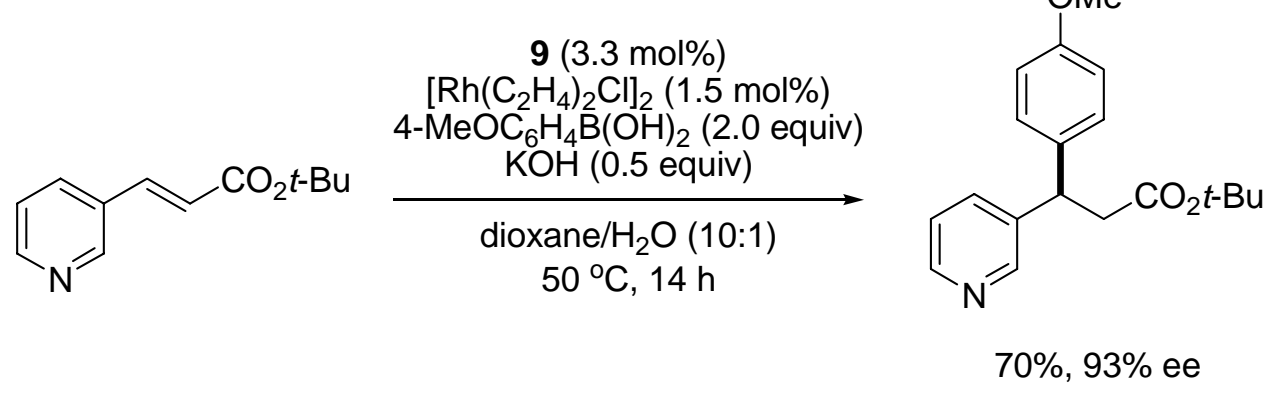

\section{(R)-tert-Butyl 3-(4-methoxyphenyl)-3-(pyridin-3-yl)propanoate}

Following the general procedure in dioxane on $0.30 \mathrm{mmol}$ scale using $t$-butyl 3-(pyridin3-yl)acrylate, 4-methoxyphenylboronic acid (1.5 equiv), and ligand 9 with a reaction time of $14 \mathrm{~h}$, the desired product (66 mg, 70\%) was isolated by flash chromatography using hexane/EtOAc (2:1) as a colorless powder. The enantioselectivity was 93\% ee (OD-H, $254 \mathrm{~nm}$, hexane:2-propanol $=92: 8$, flow rate $=0.5 \mathrm{~mL} / \mathrm{min}, \mathrm{t}_{\mathrm{r} \text { (major) }}=19.4 \mathrm{~min}, \mathrm{t}_{\mathrm{r} \text { (minor) }}=$ $21.2 \mathrm{~min}) .[\alpha]_{\mathrm{D}}{ }^{34}+6.8\left(\right.$ c $\left.1.095, \mathrm{CHCl}_{3}\right) . \mathrm{mp}=57-58^{\circ} \mathrm{C}$; IR (neat) $v=2977,2932,2835$, 1725, 1610, 1512, 1367, 1250, 1178, 1146, 1030, 833, $713 \mathrm{~cm}^{-1} ;{ }^{1} \mathrm{H}$ NMR (300 MHz, $\left.\mathrm{CDCl}_{3}\right) \delta 8.54(\mathrm{~d}, 1 \mathrm{H}, J=2.2 \mathrm{~Hz}), 8.45(\mathrm{~m}, 1 \mathrm{H}), 7.51(\mathrm{~m}, 1 \mathrm{H}), 7.20(\mathrm{~m}, 1 \mathrm{H}), 7.15$ (d, 2H, $J=8.7 \mathrm{~Hz}), 6.84(\mathrm{~d}, 2 \mathrm{H}, J=8.7 \mathrm{~Hz}), 4.45$ (t, 1H, $J=8.1 \mathrm{~Hz}), 3.77$ (s, 3H), 2.94 (d, 2H, $J$ = 8.1 Hz), $1.29(\mathrm{~s}, 9 \mathrm{H}) ;{ }^{13} \mathrm{C}$ NMR (75.5 MHz, $\left.\mathrm{CDCl}_{3}\right) \delta 170.4,158.2,149.3,147.8$, 135.0, 134.4, 128.5, 123.3, 114.0, 80.9, 55.3, 44.3, 41.9, 28.0; HRMS-EI calcd for $\mathrm{C}_{19} \mathrm{H}_{23} \mathrm{NO}_{3}[\mathrm{M}]^{+}$313.1678, found 313.1677. 

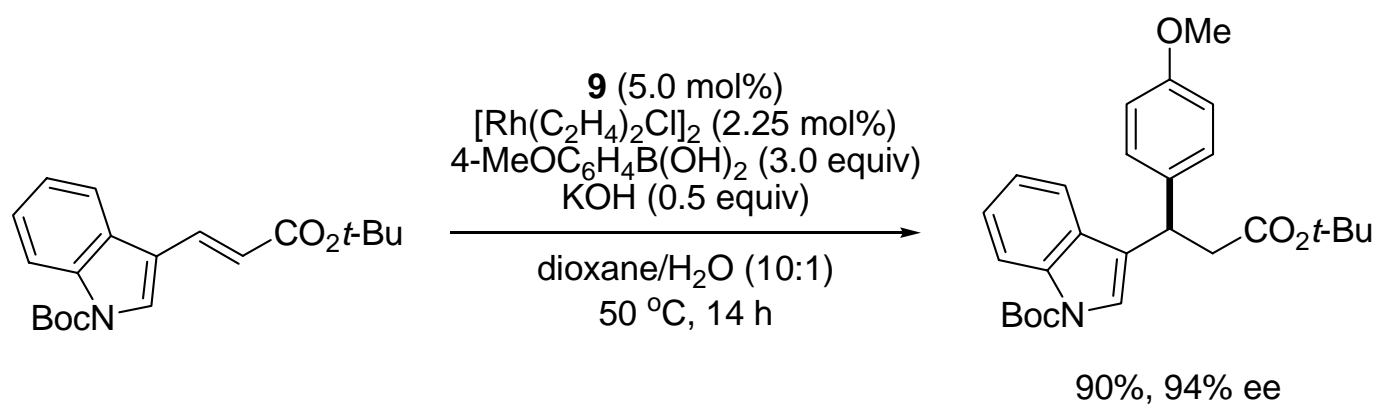

(R)-tert-Butyl

3-(3-tert-butoxy-1-(4-methoxyphenyl)-3-oxopropyl)-1H-indole-1carboxylate

Following the general procedure in dioxane on $0.20 \mathrm{mmol}$ scale using tert-butyl 3-(3-tertbutoxy-3-oxoprop-1-enyl)-1H-indole-1-carboxylate, 4-methoxyphenyl- boronic acid (3.0 equiv), $\left[\mathrm{Rh}\left(\mathrm{C}_{2} \mathrm{H}_{4}\right)_{2} \mathrm{Cl}\right]_{2}$ (2.25 mol\%), and 9 (5.0 mol\%) with a reaction time of $14 \mathrm{~h}$, the desired product (81 mg, 90\%) was isolated by flash chromatography using hexane/EtOAc (15:1) as a colorless oil. The enantioselectivity was 94\% ee (AD-H, $254 \mathrm{~nm}$, hexane:2propanol $=99: 1$, flow rate $\left.=1.0 \mathrm{~mL} / \mathrm{min}, \mathrm{t}_{\mathrm{r}(\text { major })}=9.9 \mathrm{~min}, \mathrm{t}_{\mathrm{r}(\text { minor })}=16.6 \mathrm{~min}\right):[\alpha]_{\mathrm{D}}{ }^{34}$ -81.5 (c 0.435, $\mathrm{CHCl}_{3}$ ). IR (neat) $v=2977,2932,2835,1731,1610,1511,1453,1369$, 1253, 1157, 1092, 1036, 912, $746 \mathrm{~cm}^{-1} ;{ }^{1} \mathrm{H}$ NMR $\left(300 \mathrm{MHz}, \mathrm{CDCl}_{3}\right) \delta 8.08(\mathrm{~d}, 1 \mathrm{H}, J=$ 8.0 Hz), 7.47 (s, 1H), 7.31-7.19 (m, 2H), 7.22 (d, 2H, $J=8.8 \mathrm{~Hz}), 7.10$ (m, 1H), 6.80 (d, 2H, $J=8.8 \mathrm{~Hz}$ ), 4.60 (t, 1H, $J=7.7 \mathrm{~Hz}$ ), 3.76 (s, 3H), 3.02 (dd, 1H, $J=15.3,7.7 \mathrm{~Hz}$ ), 2.85 (dd, $1 \mathrm{H}, J=15.3,7.7 \mathrm{~Hz}), 1.67$ (s, 9H), 1.33 (s, 9H); ${ }^{13} \mathrm{C}$ NMR (75.5 MHz, $\mathrm{CDCl}_{3}$ ) $\delta 170.9,158.1,149.7,135.6,134.5,129.7,128.7,124.3,123.4,122.3,122.0,119.8$, 115.1, 113.8, 83.5, 80.6, 55.3, 42.4, 38.5, 28.4, 28.1; HRMS-MALDI calcd for $\mathrm{C}_{27} \mathrm{H}_{33} \mathrm{NO}_{5} \mathrm{Na}[\mathrm{M}]^{+}$474.2251, found 474.2244. 
<smiles>CC(C)(C)OC(=O)CC(c1ccccc1)c1ccccc1[N+](=O)[O-]</smiles>

10

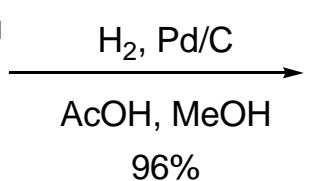

$96 \%$

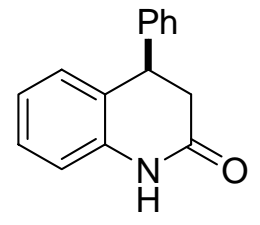

11

\section{(R)-4-Phenyl-3,4-dihydroquinolin-2(1H)-one (11)}

A mixture of 10 (75 mg, $0.23 \mathrm{mmol}$ ) and Pd/C (24 mg, $0.023 \mathrm{mmol}, 10 \mathrm{wt} \% \mathrm{Pd}$ on C) under $\mathrm{Ar}$ was suspended in $\mathrm{MeOH}(2.0 \mathrm{~mL})$ and treated with $\mathrm{AcOH}(13 \mu \mathrm{L}, 0.23 \mathrm{mmol})$. The flask was evacuated and purged with $\mathrm{H}_{2}(1 \mathrm{~atm})$ and the reaction was vigorously stirred for $1.5 \mathrm{~h}$. The $\mathrm{H}_{2}$ balloon was removed and the reaction was stirred under Ar for $14 \mathrm{~h}$, filtered through Celite and concentrated. The residue was purified by chromatography on $\mathrm{SiO}_{2}(1: 1$, hexanes/EtOAc) to give 11 (49 mg, 96\%) as a colorless solid: $[\alpha]_{\mathrm{D}}^{27}-48.8\left(c\right.$ 0.79, $\left.\mathrm{CHCl}_{3}\right)$. IR (neat) $v=3208,3059,2914,1679,1593,1486$, 1374, $1260 \mathrm{~cm}^{-1}$; ${ }^{1} \mathrm{H}$ NMR (300 MHz, $\left.\mathrm{CDCl}_{3}\right) \delta 9.11$ (bs, $\left.1 \mathrm{H}\right)$, 7.37-7.19 (m, $\left.6 \mathrm{H}\right)$, 6.996.89 (m, $3 \mathrm{H}), 4.31(\mathrm{t}, 1 \mathrm{H}, J=7.5 \mathrm{~Hz}), 3.02-2.88(\mathrm{~m}, 2 \mathrm{H}) ;{ }^{13} \mathrm{C}$ NMR $(75.5 \mathrm{MHz}$, $\left.\mathrm{CDCl}_{3}\right) \delta 170.8,141.3,136.9,128.8,128.2,127.9,127.7,127.1,126.5,123.2,115.6$ 42.0, 38.5; HRMS-EI calcd for $\mathrm{C}_{15} \mathrm{H}_{13} \mathrm{NO}[\mathrm{M}]^{+}$223.0992, found 223.0992 . 

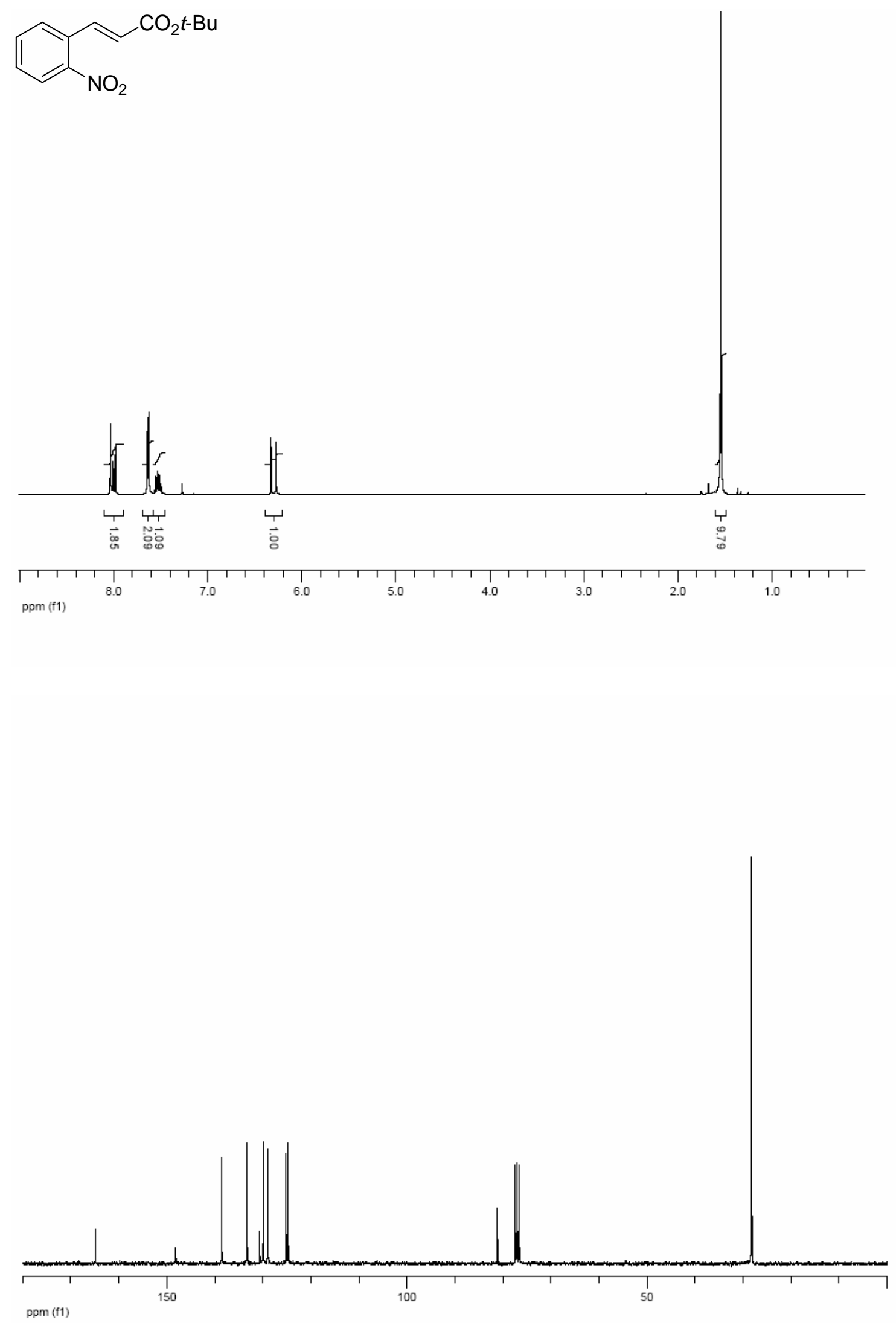

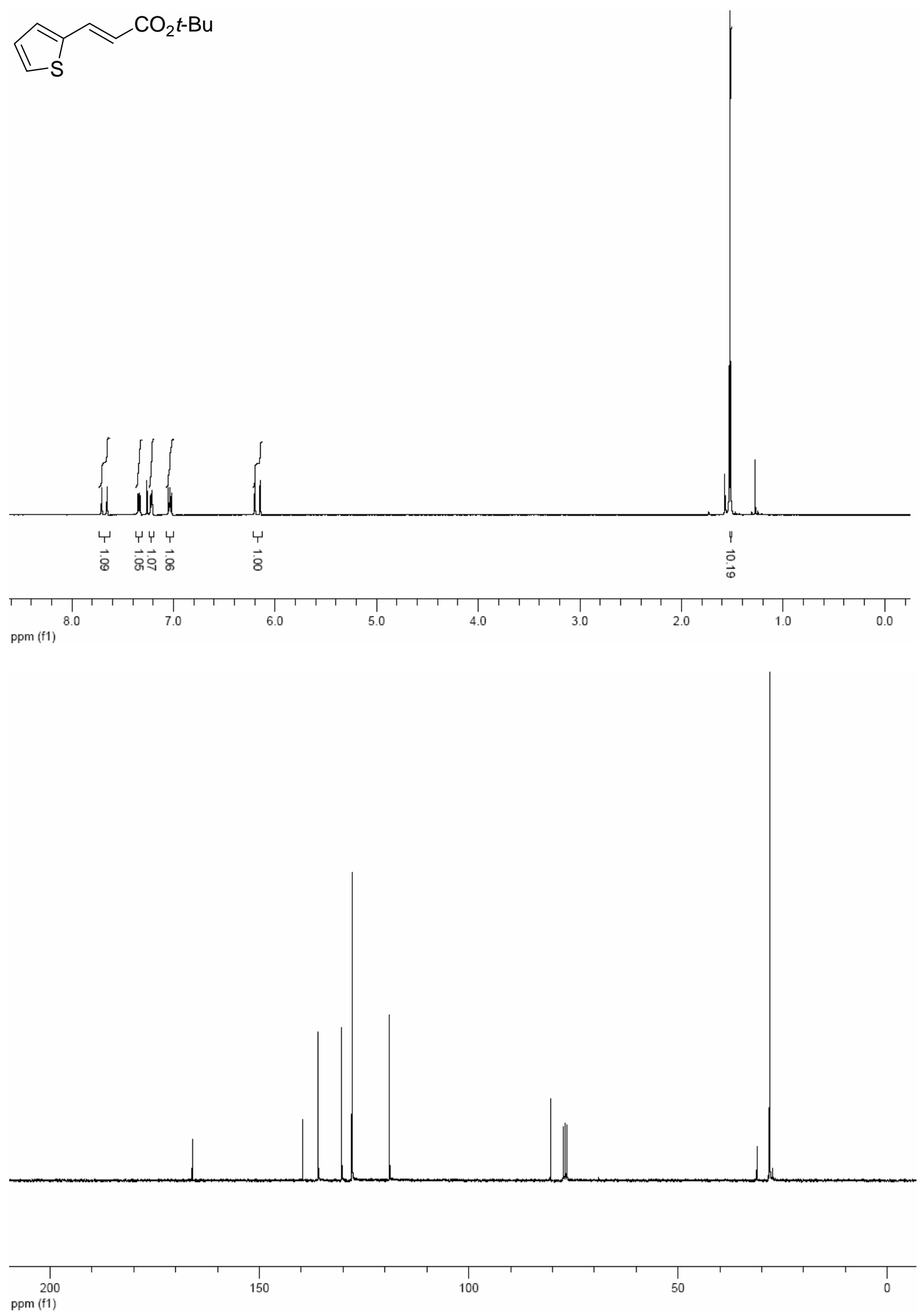
S $\mathrm{CO}_{2} t-\mathrm{Bu}$
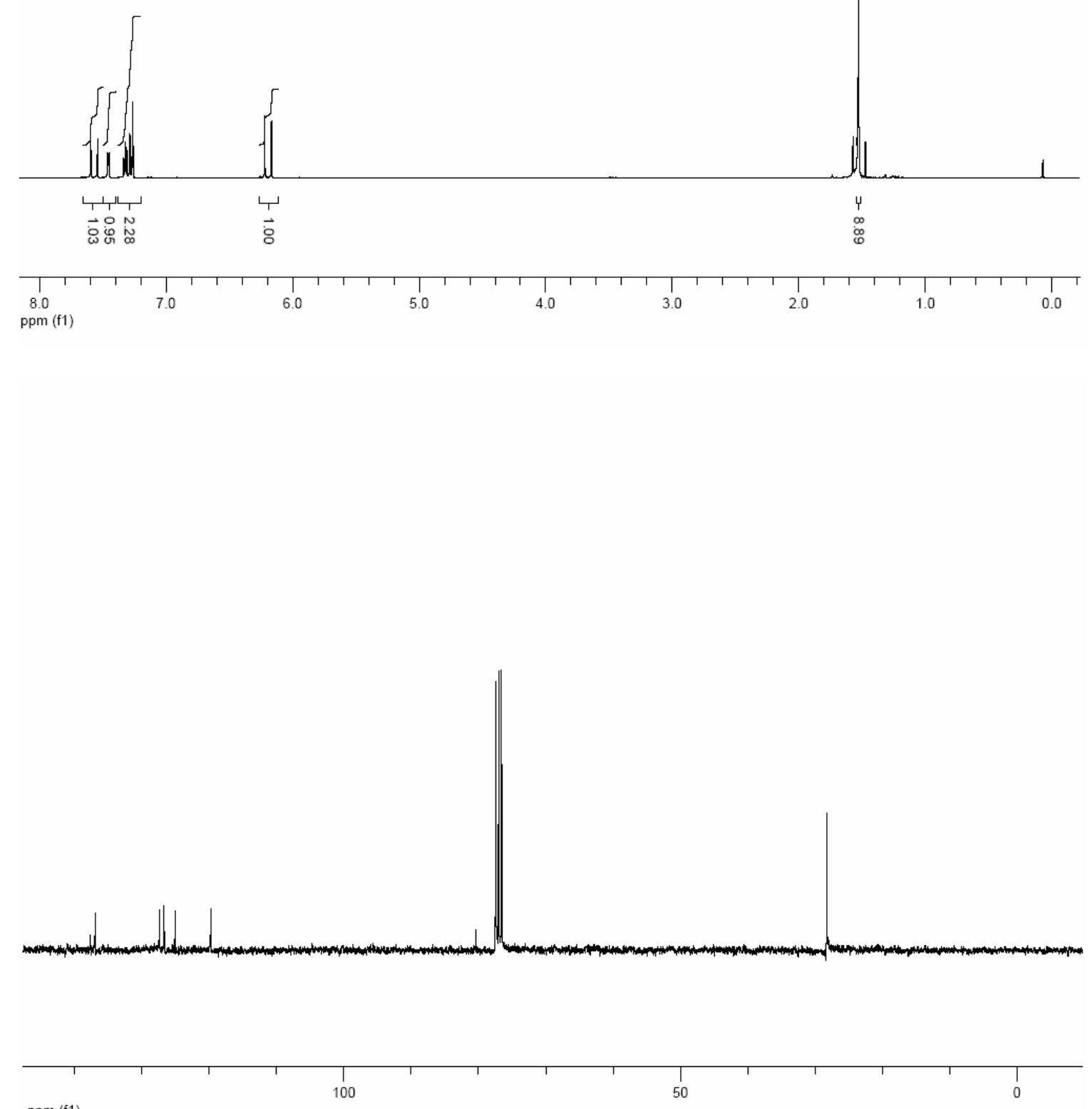

ppm (f1) 

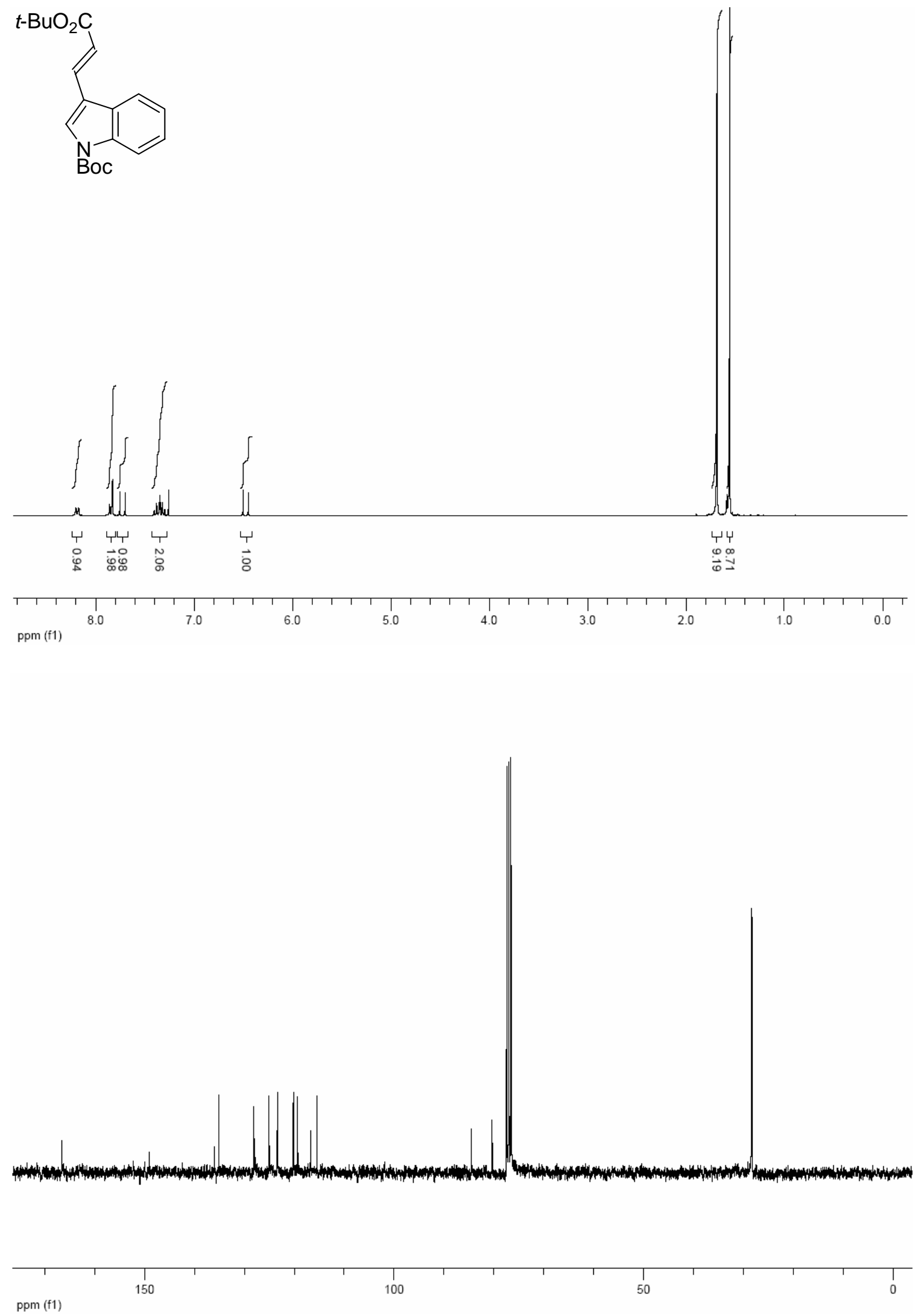
<smiles>CCOC(=O)CC(c1ccccc1)c1ccc(OC)cc1</smiles>

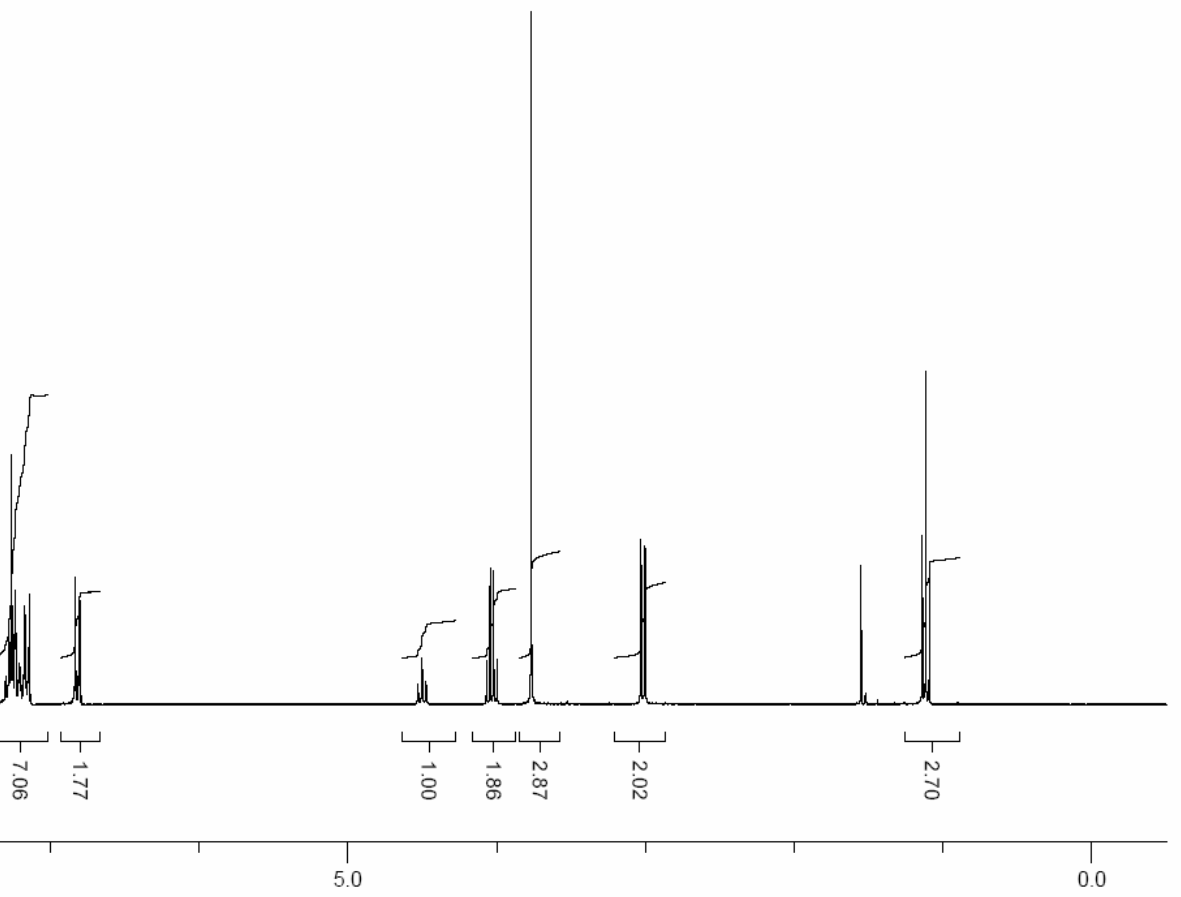

ppm (f1)
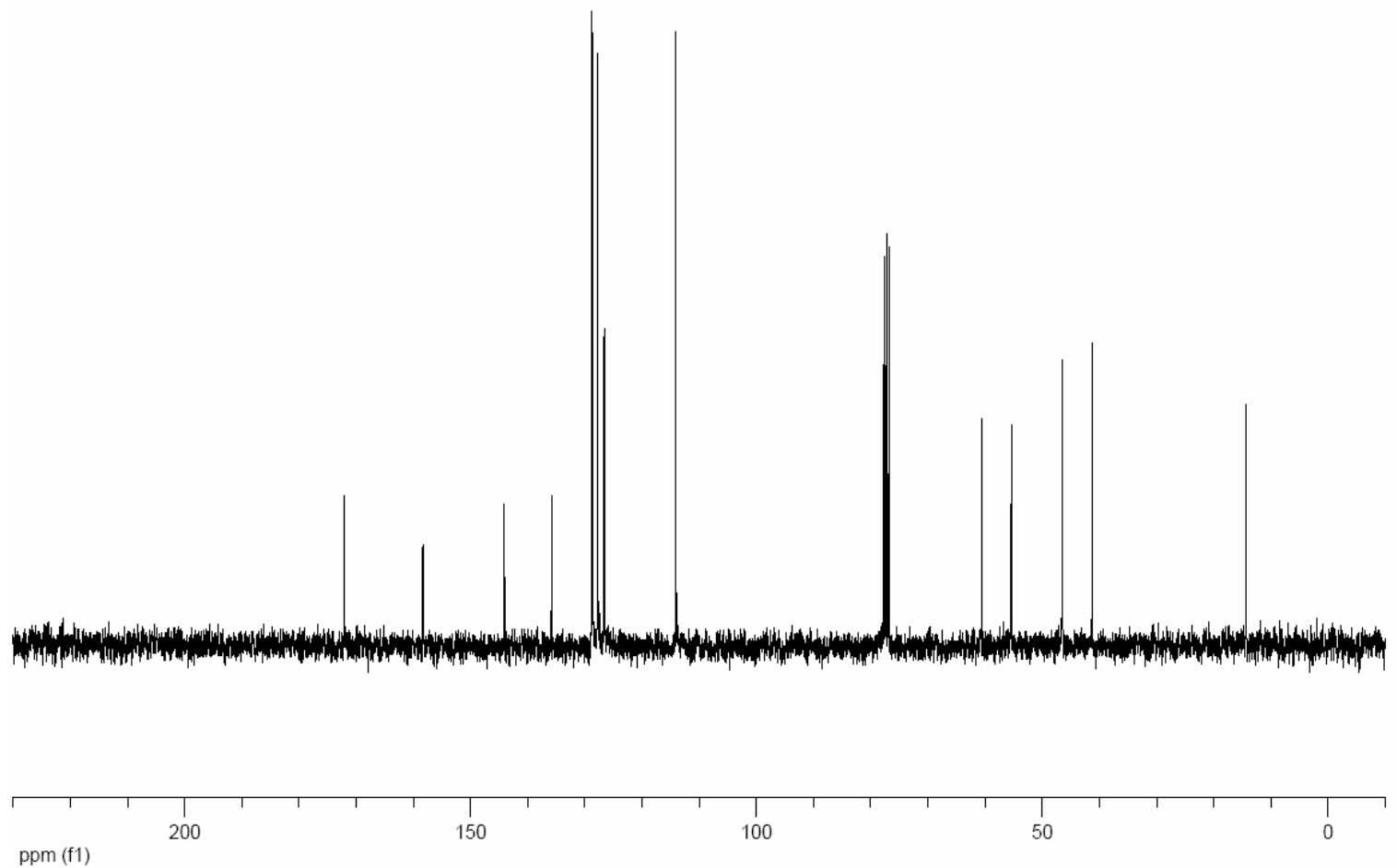
<smiles>COc1ccc(C(CC(=O)OCc2ccccc2)c2ccccc2)cc1</smiles>

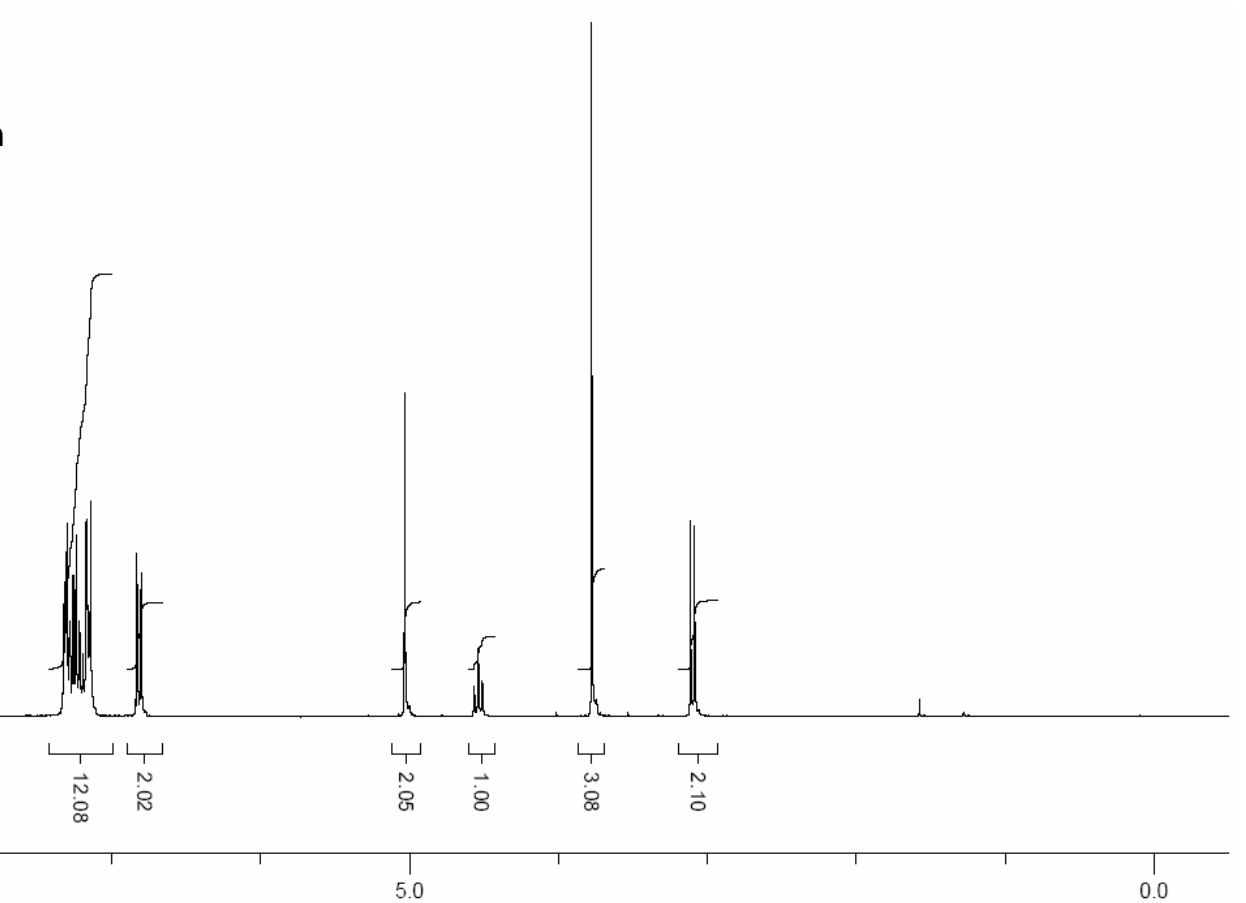

ppm (f1)

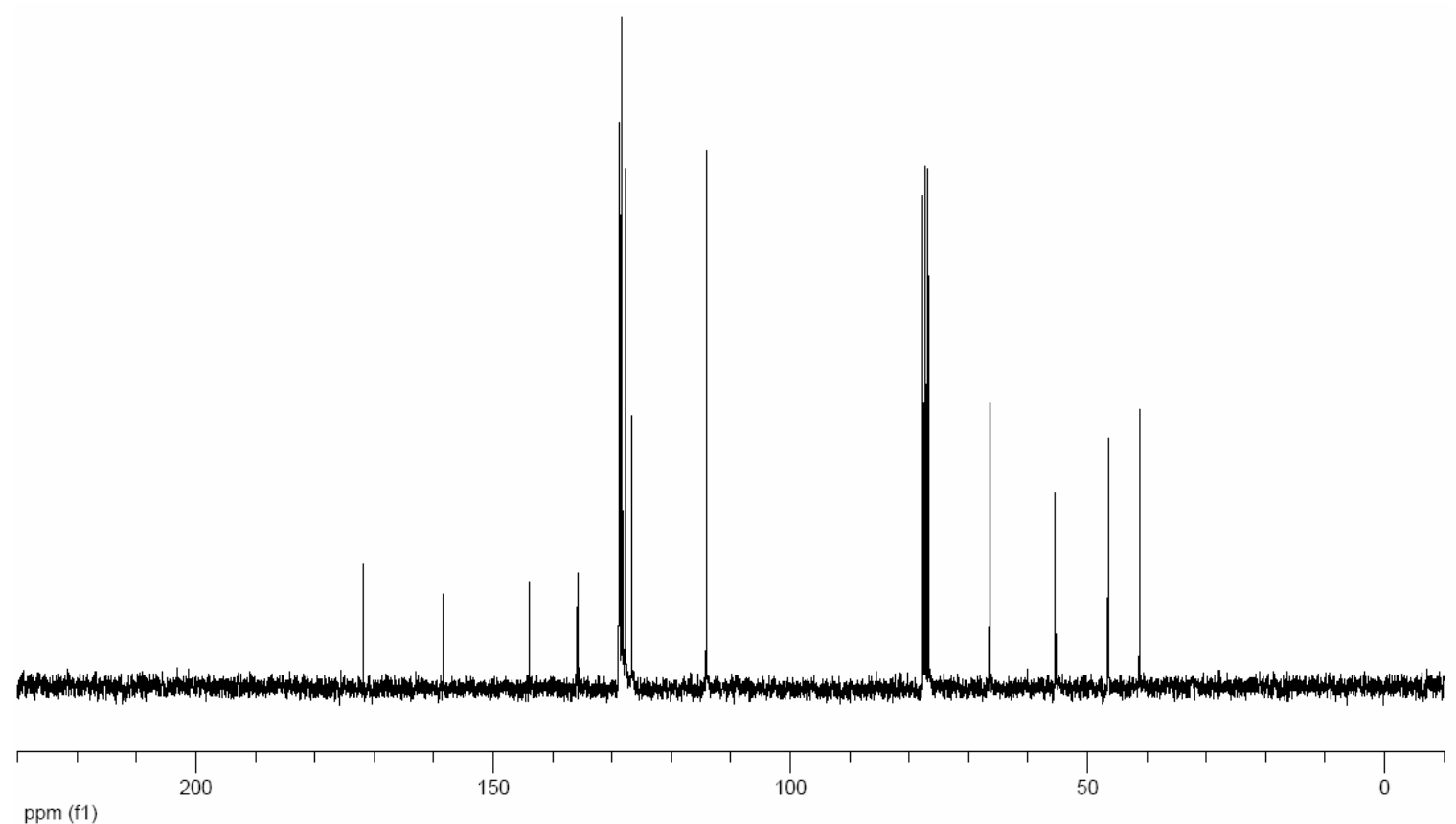


<smiles>COc1ccc(C(CC(=O)OC(C)(C)C)c2ccccc2)cc1</smiles>

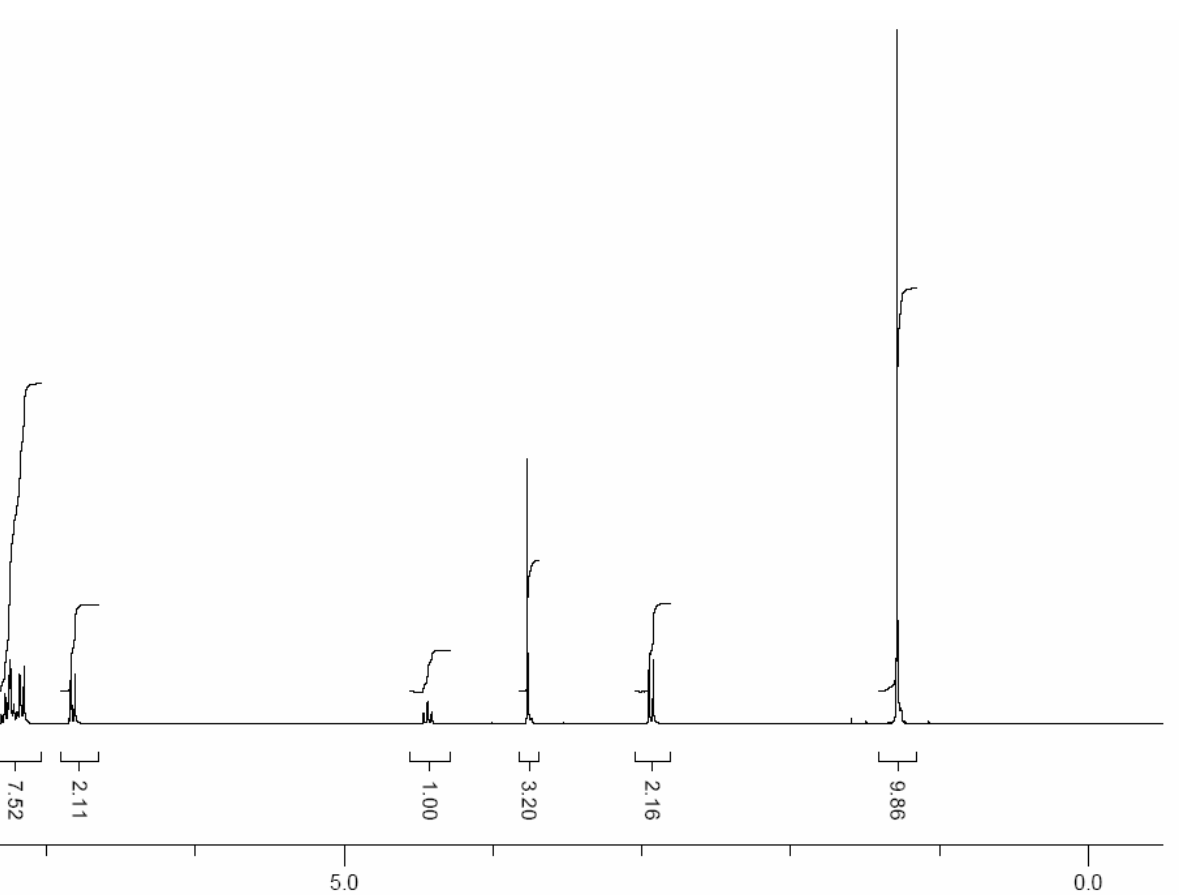

ppm (f1)

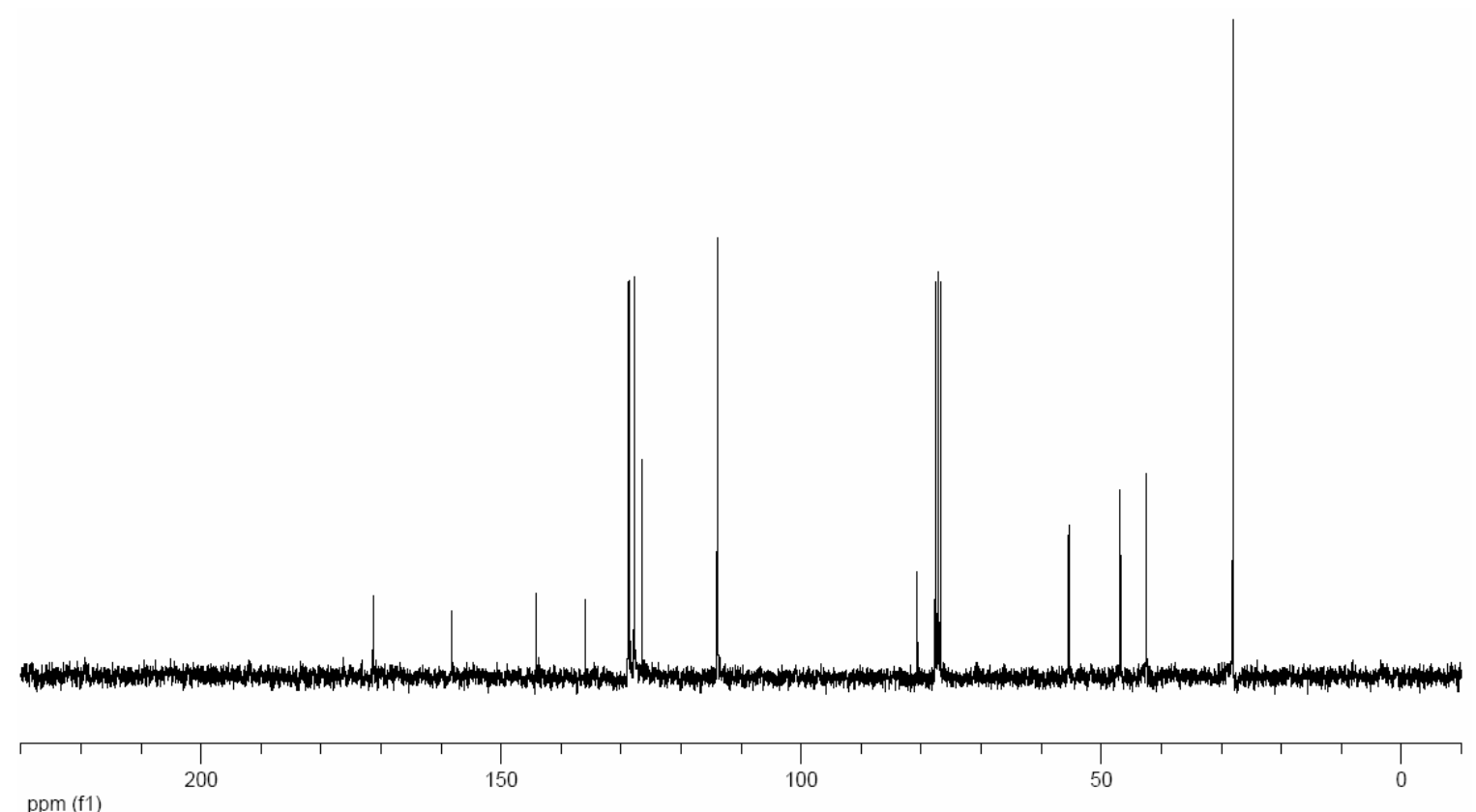



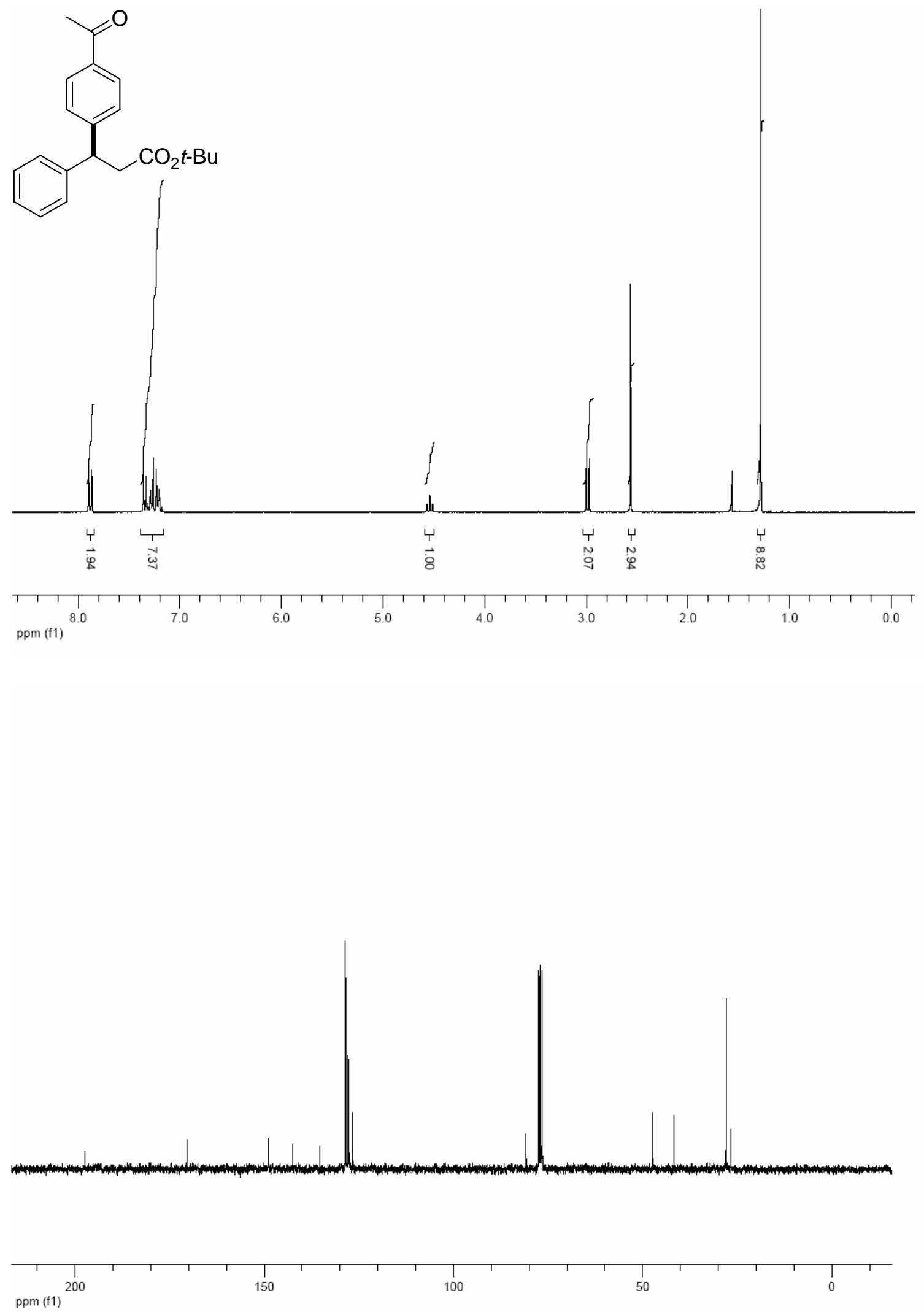
<smiles>CCC(C)OC(=O)CC(c1ccccc1)c1cccc(C(F)(F)F)c1</smiles>
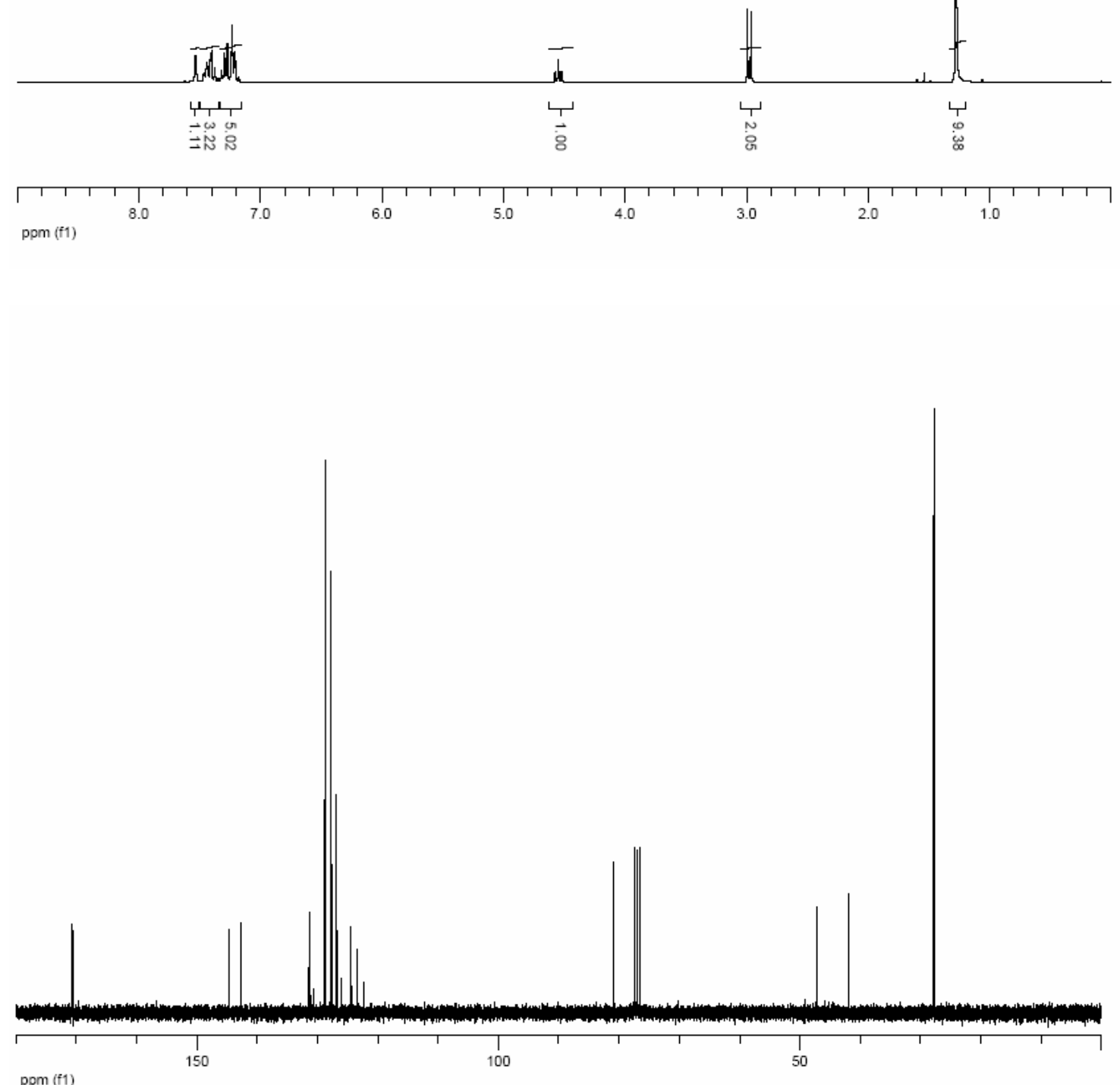
<smiles>CC(C)(C)OC(=O)CC(c1ccccc1)c1cccc(F)c1</smiles>
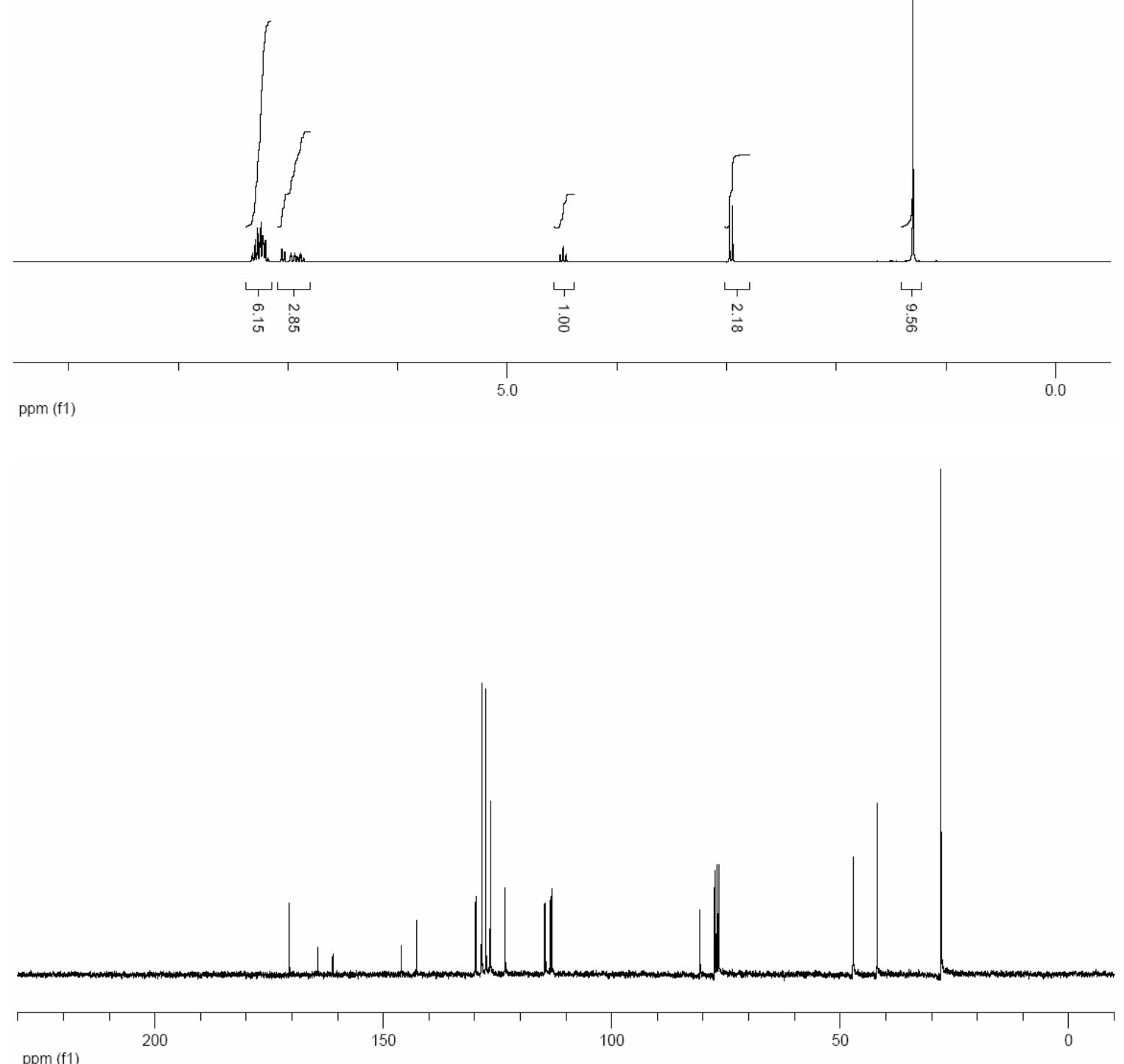
<smiles>COc1ccc(C(CC(=O)OC(C)(C)C)c2cccc(F)c2)cc1</smiles>

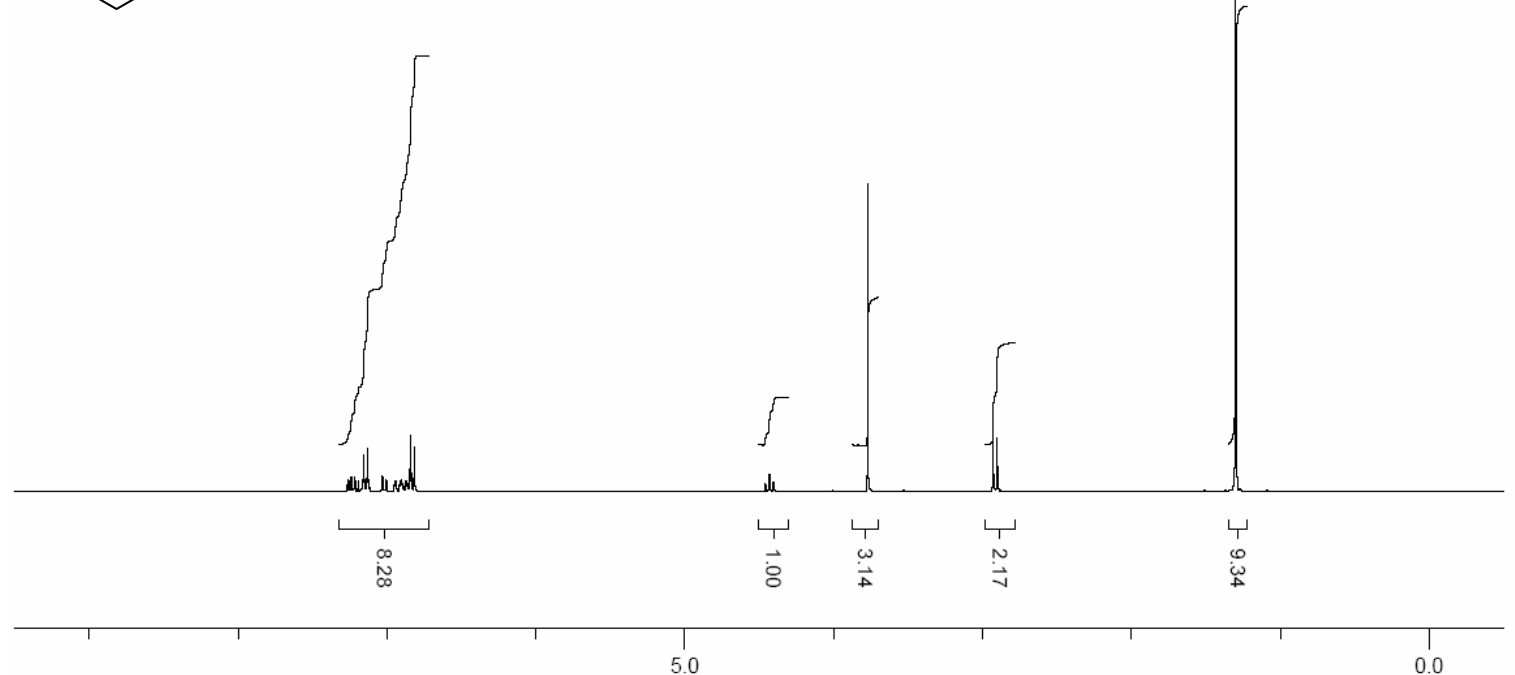

ppm (f1)

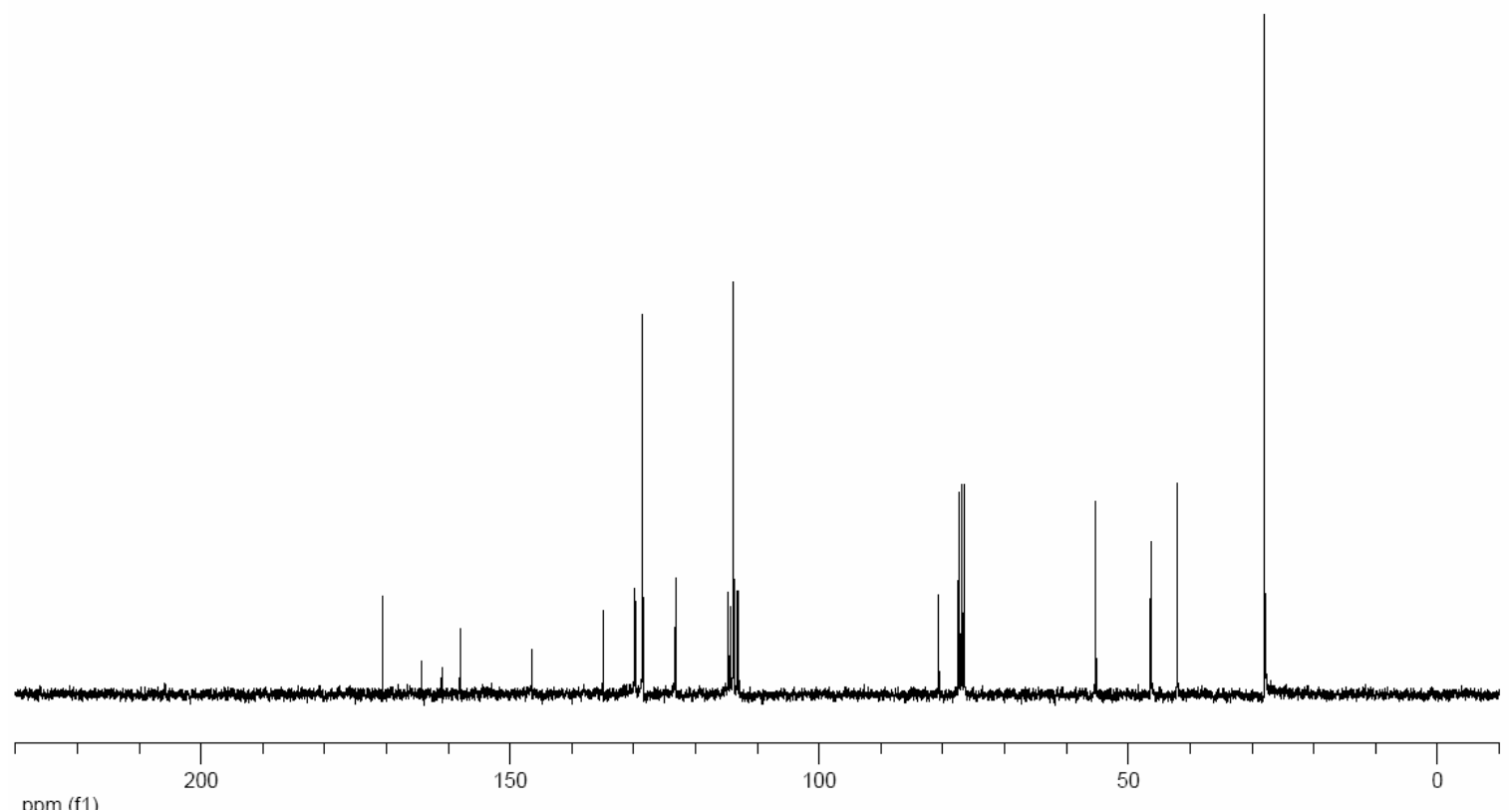



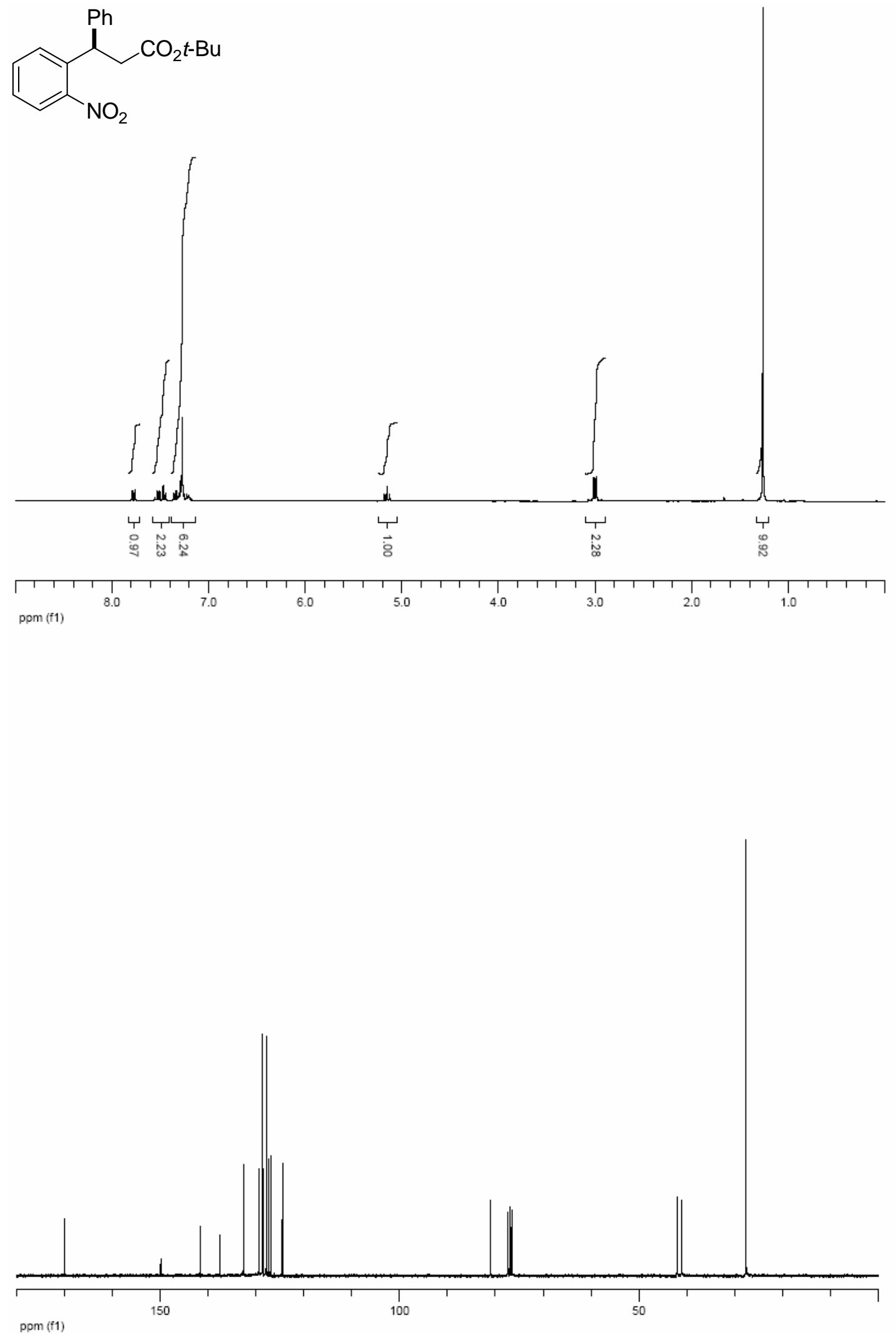

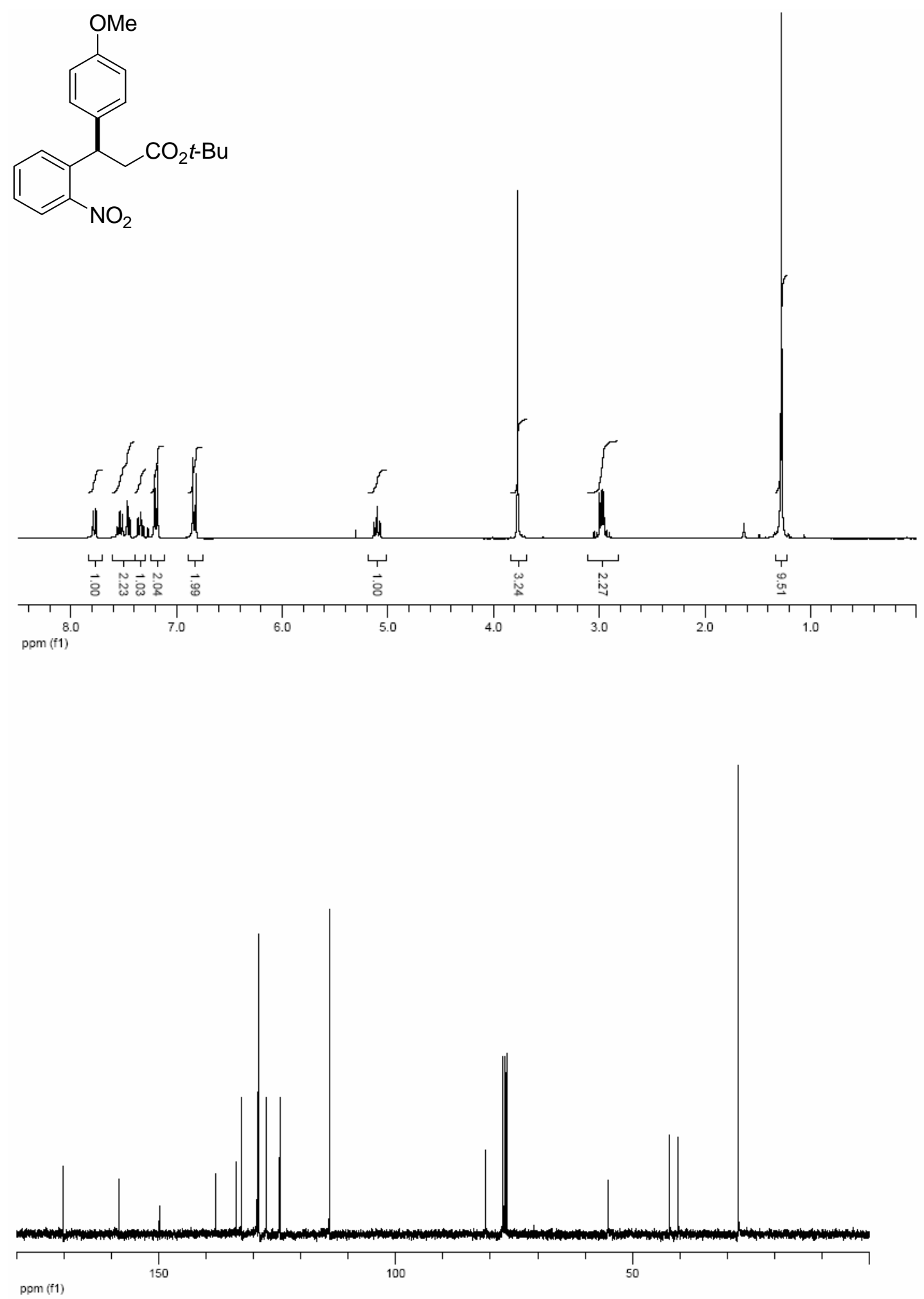
<smiles>COc1ccc(C(CC(=O)OC(C)(C)C)c2ccco2)cc1</smiles>

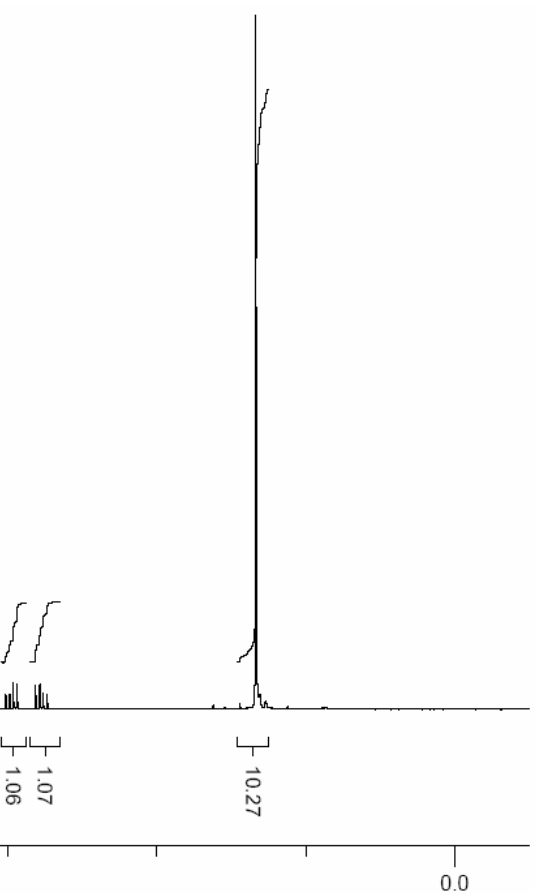

ppm (f1)

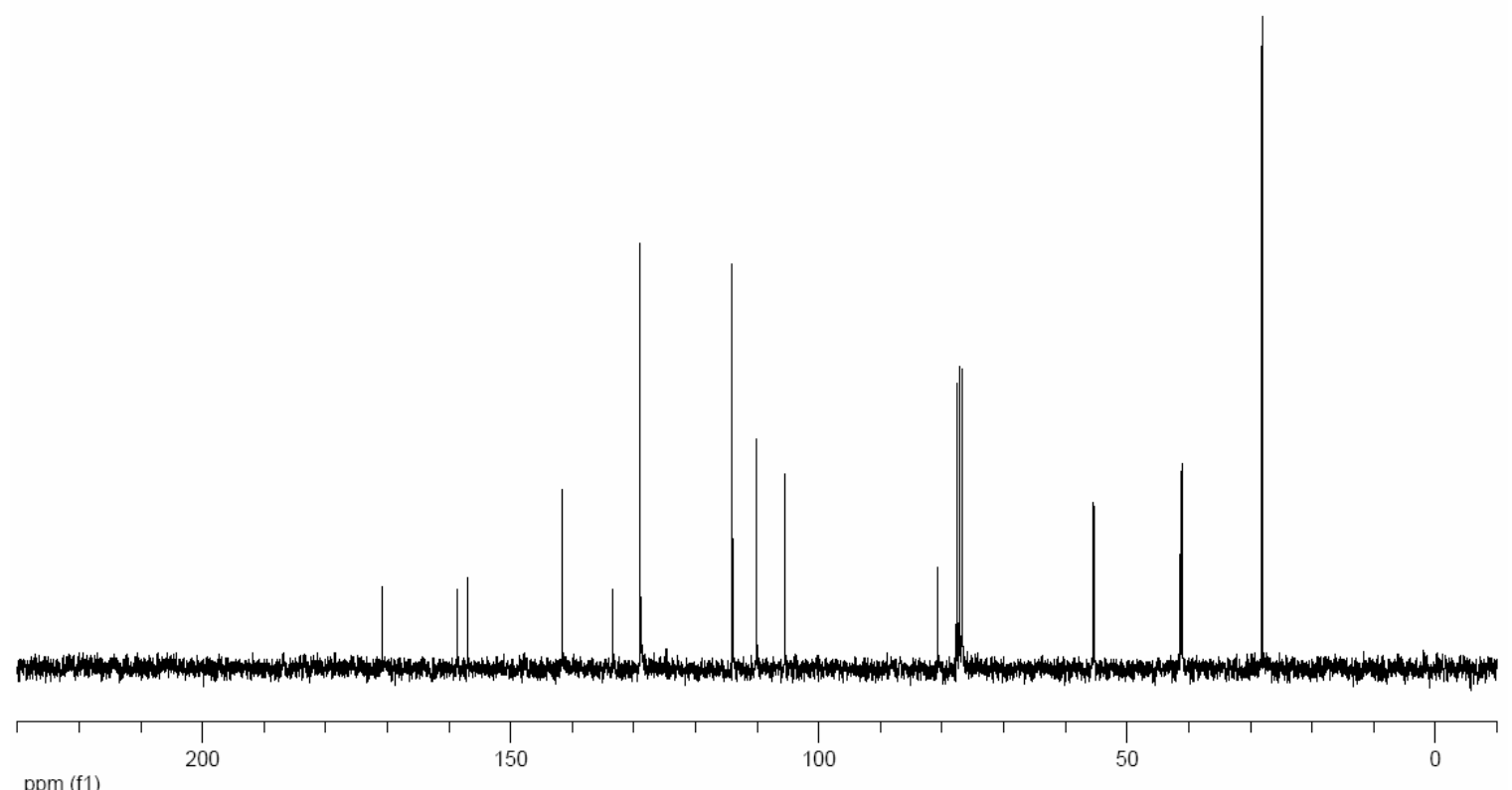


<smiles>CC(=O)OCC(c1ccc(C(C)(C)C)cc1)c1ccco1</smiles>

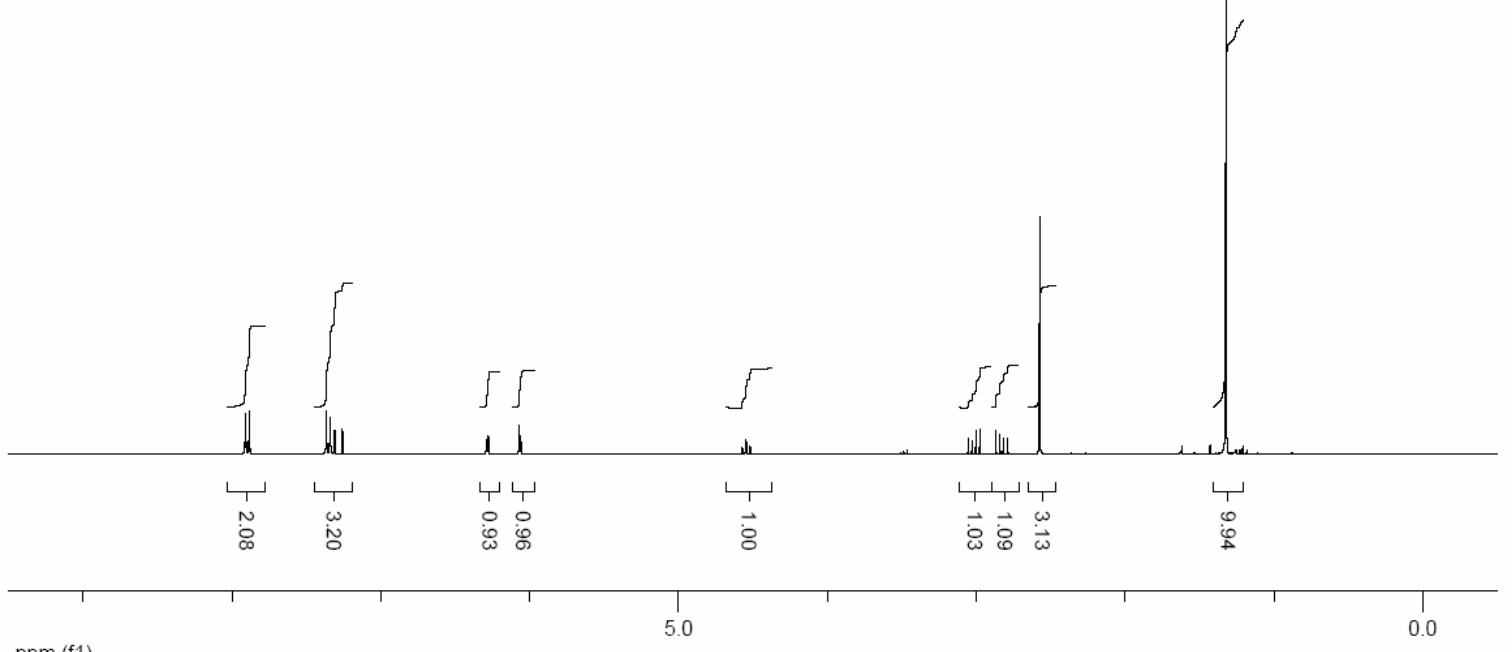
ppm (f1)

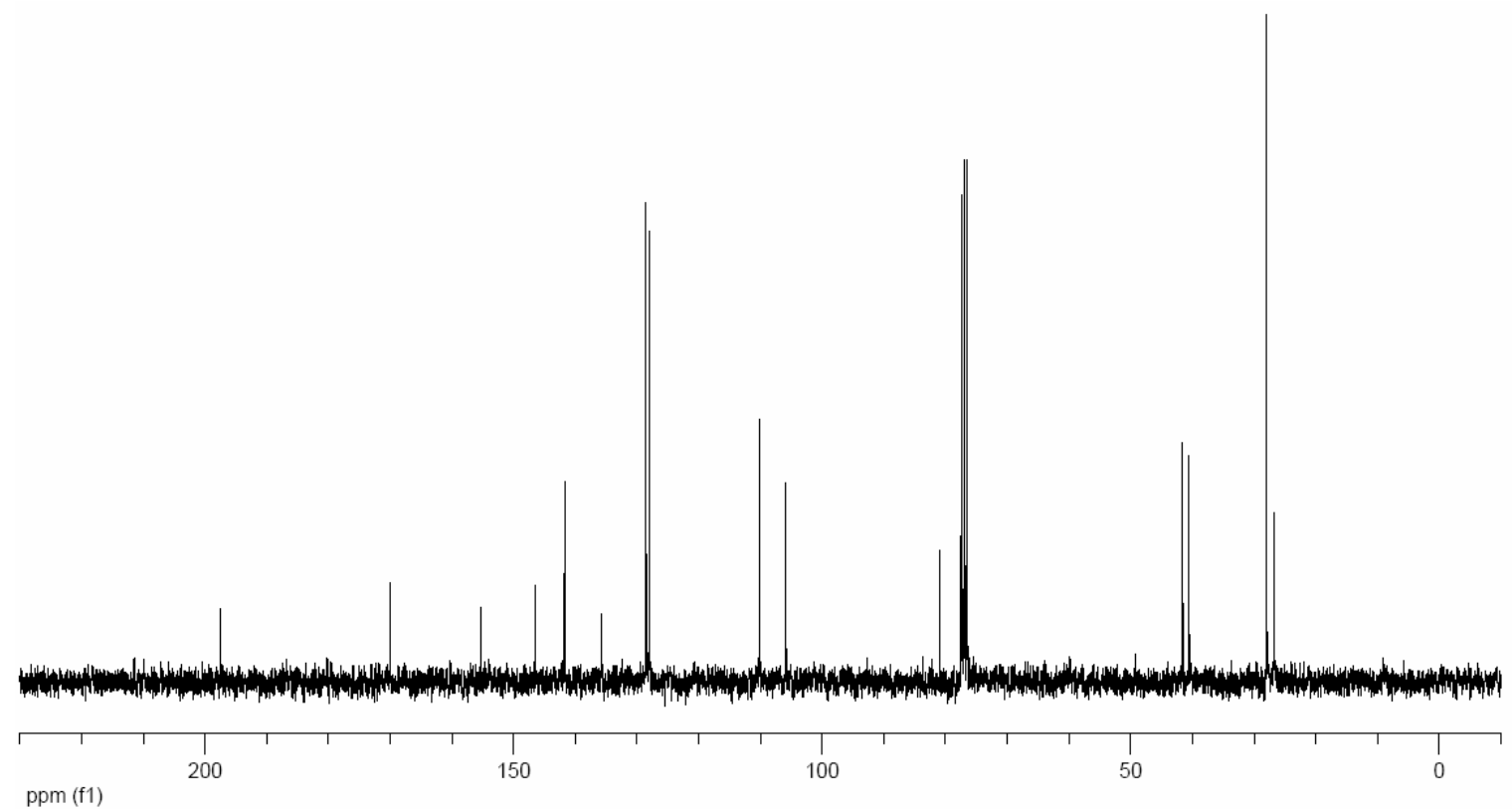


<smiles>CC(=O)c1ccc(C(CC(=O)OC(C)(C)C)c2ccsc2)cc1</smiles>
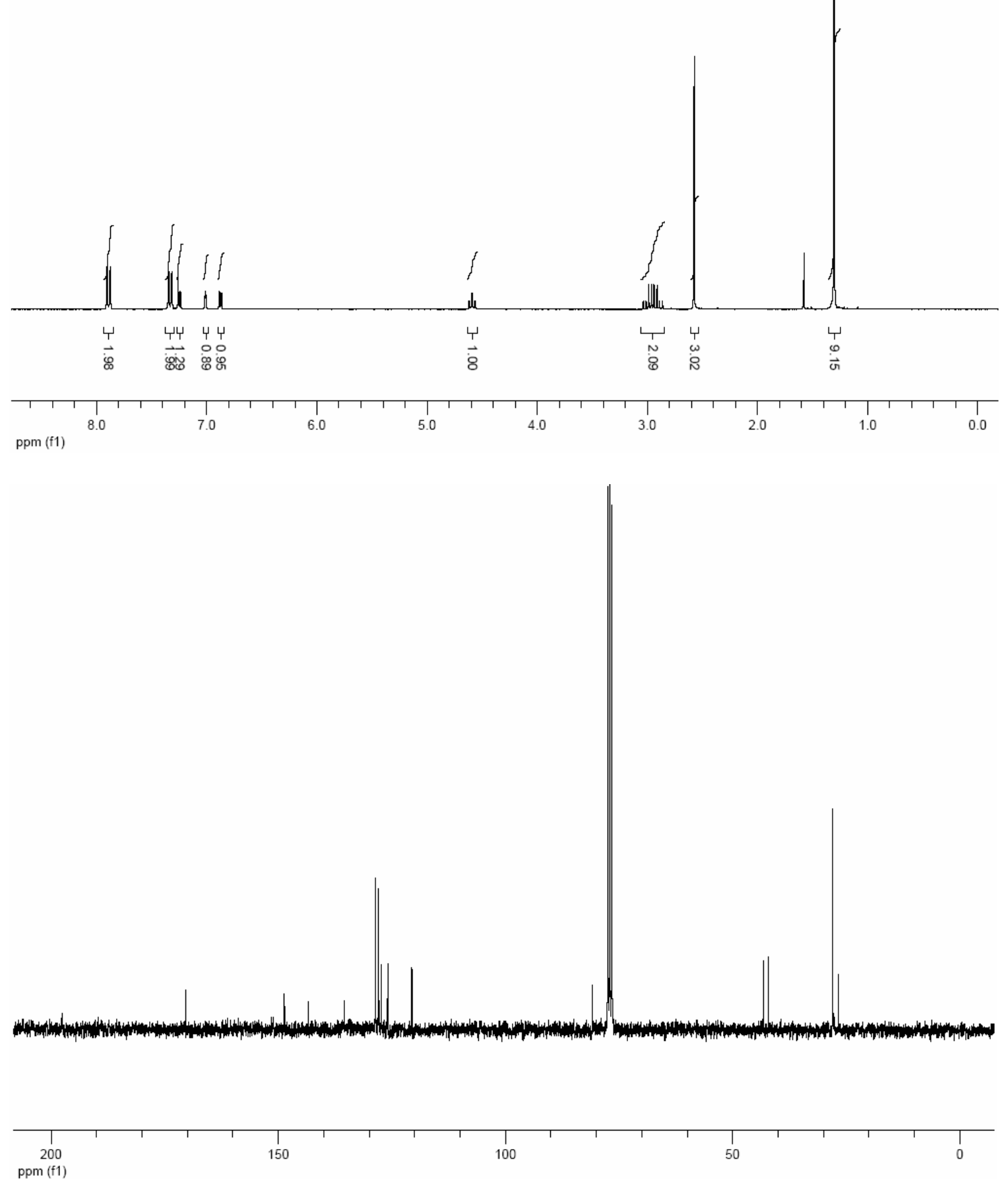
<smiles>CC(=O)c1ccc(C(CC(=O)OC(C)(C)C)c2cccs2)cc1</smiles>

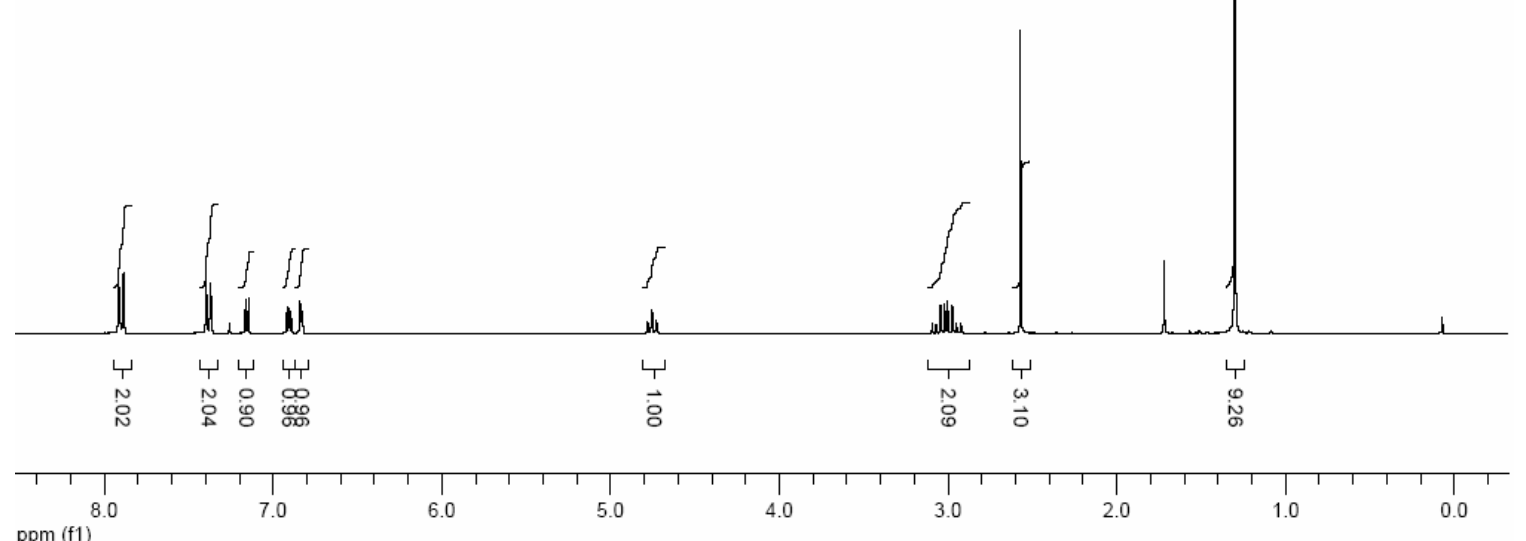
ppm (f1)
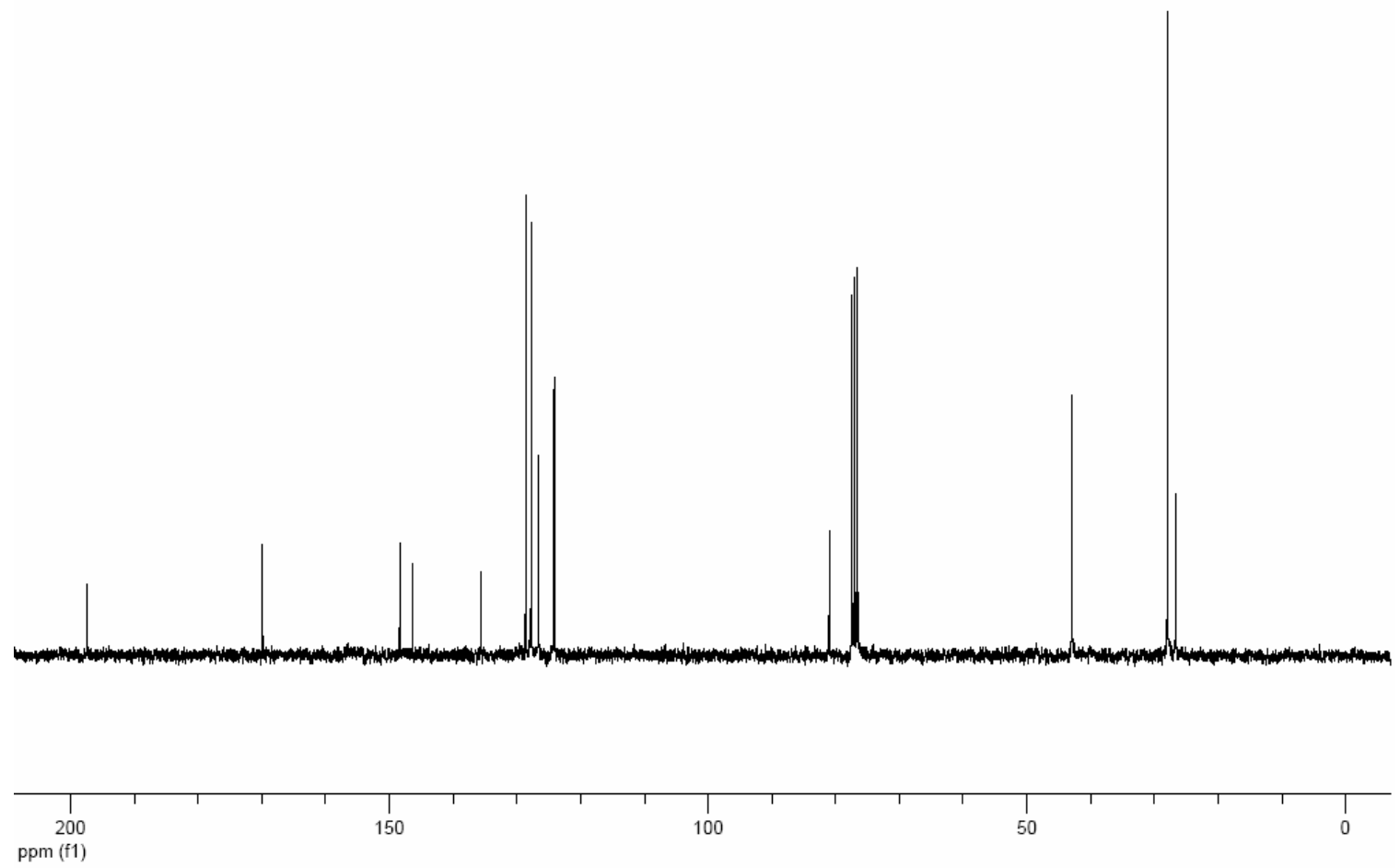
<smiles>COc1ccc(C(CC(=O)OC(C)(C)C)c2cccnc2)cc1</smiles>

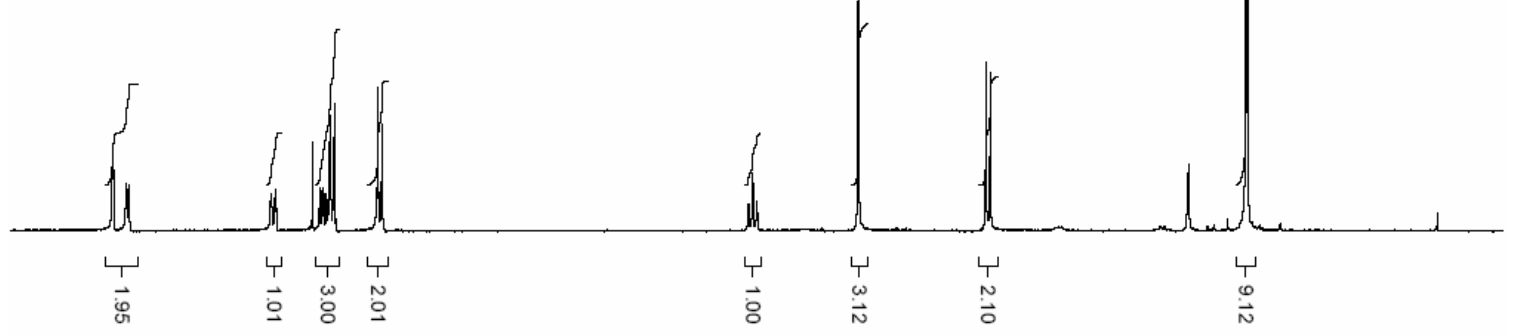

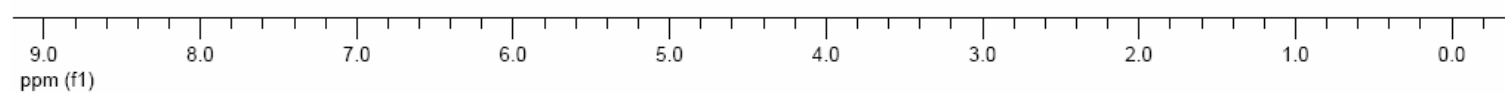
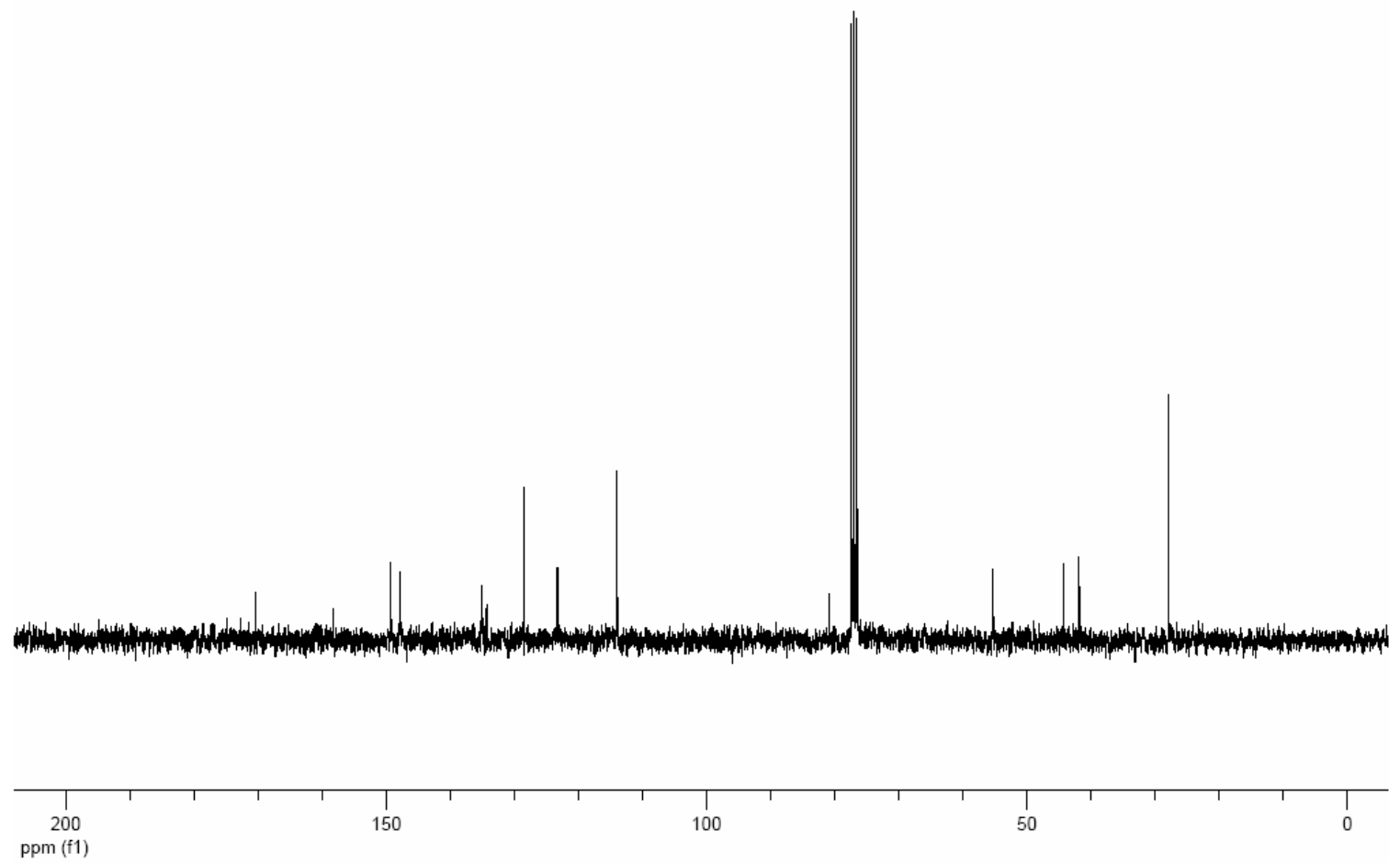
<smiles>COc1ccc(C(CC(=O)OCC(C)(C)C)c2cn(C(C)(C)C)c3ccccc23)cc1</smiles>
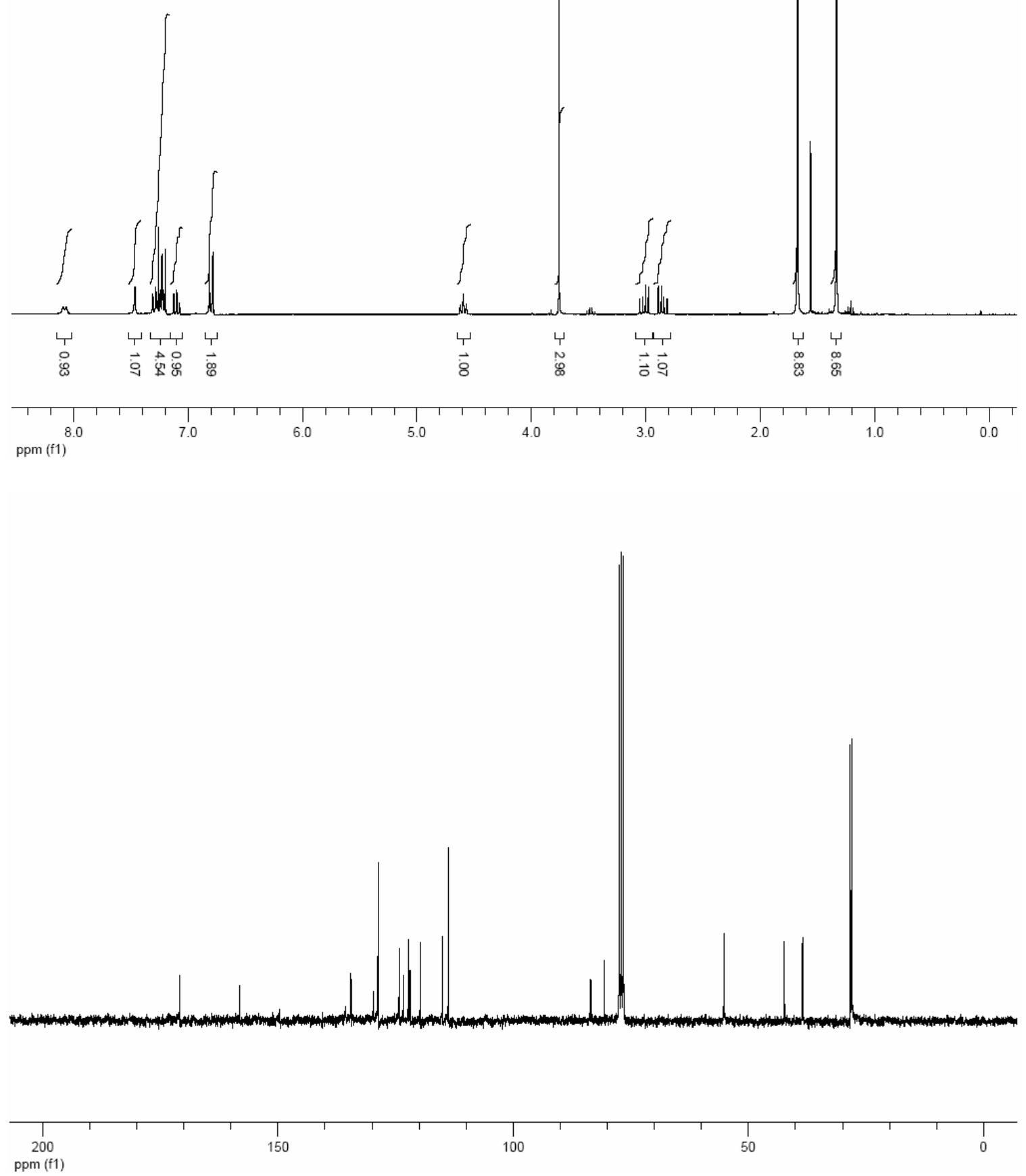


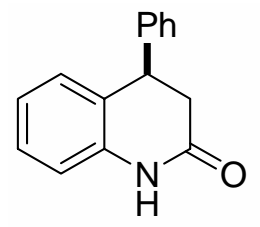

11
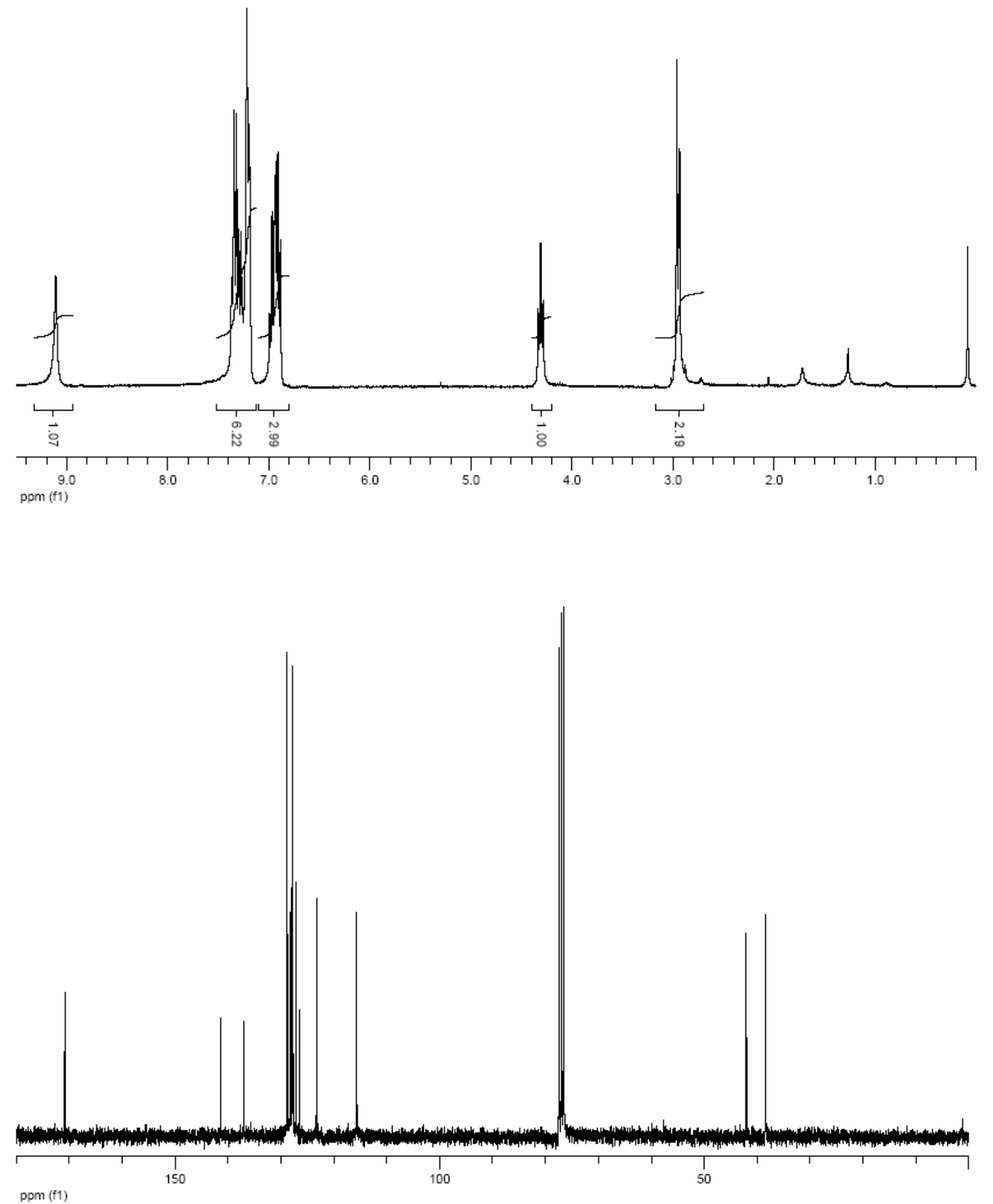